\title{
Theory of hydrogen diffusion and reactions in crystalline silicon
}

\author{
Chris G. Van de Walle, ${ }^{*}$ P. J. H. Denteneer, Y. Bar-Yam, and S. T. Pantelides \\ IBM Research Division, Thomas J. Watson Research Center, Yorktown Heights, New York 10598
}

(Received 21 November 1988)

\begin{abstract}
The behavior of hydrogen in crystalline silicon is examined with state-of-the-art theoretical techniques, based on the pseudopotential-density-functional method in a supercell geometry. Stable sites, migration paths, and barriers for different charge states are explored and displayed in totalenergy surfaces that provide immediate insight into these properties. The bond-center site is the global minimum for the neutral and positive charge states; in the negative charge state, the tetrahedral interstitial site is preferred. The positive charge state is energetically favorable in $p$-type material, providing a mechanism for passivation of shallow acceptors: electrons from the $\mathrm{H}$ atoms annihilate the free holes, and formation of $\mathrm{H}$-acceptor pairs follows compensation. Also addressed are the issues of molecule formation and hydrogen-induced damage. A number of different mechanisms for defect formation are examined; hydrogen-assisted vacancy formation is found to be an exothermic process.
\end{abstract}

\section{INTRODUCTION}

The topic of hydrogen $(\mathrm{H})$ in semiconductors has recently attracted a great deal of interest. From a fundamental point of view, it is attractive to study the interaction between $\mathrm{H}$, the most elementary atom, and silicon (Si), the prototypical semiconductor. The role of $\mathrm{H}$ in crystalline semiconductors has also emerged as an important technological problem: its effects have recently most dramatically been observed in the passivation of shallow impurities. In this paper we will concentrate on the behavior of hydrogen itself as it diffuses through a Si crystal. The information about stable sites and charge states obtained here is essential for understanding not only isolated hydrogen, but also its reactions with other impurities.

Hydrogen has been known for a long time to saturate dangling bonds at surfaces, vacancies, and grain boundaries, and to passivate deep-level defects, such as those due to transition-metal impurities. ${ }^{1}$ In cases where deep levels are detrimental for device properties, their elimination by hydrogenation is of great benefit. The fact that hydrogen can also passivate shallow impurities has only been appreciated more recently. ${ }^{1-9}$ Shallow levels determine the doping of the semiconductor, which determines its characteristics in device operation; accidental passivation of these impurities can cause outright failure of the device. On the other hand, one can envision applications in which intentional passivation of certain areas of a device could be an integral part of the fabrication process. Since hydrogen is present, intentionally or not, during many of the processing steps for fabricating modern semiconductor devices, its potential effects, whether harmful or beneficial, should be thoroughly understood.

There exist indeed a wide variety of ways in which $\mathrm{H}$ can penetrate $\mathrm{Si}$, a number of which are discussed in Ref. 10. They include crystal growth, high-temperature permeation, ${ }^{11}$ ion implantation, ${ }^{12}$ chemomechanical polishing, ${ }^{13}$ wet etching, unintentional hydrogenation during ion bombardment, plasma etching, boiling in water, and surface exposure to monatomic $H$. The latter can be achieved by placing the sample in or downstream from a microwave plasma. ${ }^{4,14}$ If the sample is shielded from the plasma, this is the preferred way for introducing $\mathbf{H}$ under the best controlled conditions, avoiding any additional damage in the material.

Passivation of the electrical activity of $p$-type silicon was first observed in metal-oxide-semiconductor (MOS) capacitors by Sah et al. ${ }^{2}$ who suggested $\mathrm{H}$ as the probable cause. Subsequent experiments by Pankove et al. ${ }^{3}$ and by Johnson ${ }^{4}$ unambiguously showed the correlation between $H$ and acceptor profiles, and established the existence of $\mathrm{H}$-acceptor pairs. Initially, passivation of shallow donors was thought to be nonexistent ${ }^{2,3}$ or very weak. ${ }^{5}$ Recently, however, conclusive evidence has been provided for passivation of samples doped with $\mathbf{P}$, As, and $\mathrm{Sb}$, in which a reduction of up to $80 \%$ was observed in carrier concentrations. ${ }^{6}$ This passivation, while dramatic, is still not as complete as can be obtained in $p$ type samples.

Apart from its role in interacting with existing defects and impurities, hydrogen has recently been shown to induce defects as well. ${ }^{15}$ Extended defects (described as "platelets") in the near-surface region were observed after hydrogenation, and correlated with the presence of large concentrations of $\mathrm{H}$.

A number of authors ${ }^{2-5,7,9}$ have offered interpretations of the passivation data seeking to unravel the underlying mechanisms. Attempts to explain the observed phenomena led to a number of contradictory assumptions regarding the nature of the charge states of $\mathbf{H}$ along its diffusion path, and hence about the $\mathrm{H}$-impurity reactions that can occur. Particular models were advanced for the structure of the hydrogen-impurity complexes that are a result of passivation. The electronic structure of these complexes is such that all impurity levels are removed from the band gap. A complete understanding of the passivation process can only be obtained, however, by considering 
the reactions that lead to $\mathrm{H}$-impurity pairing. This requires a description of the behavior of $\mathrm{H}$ as an isolated impurity, an aspect that was first stressed in a paper by one of the present authors. ${ }^{16}$ On the basis of available data, it was proposed that $\mathrm{H}$ has a donor level in the band gap. Accordingly, passivation of $p$-type material is due to compensation, i.e., the electron from the $\mathrm{H}$ annihilates a free hole, and $\mathrm{H}^{+}$is formed. Pairing of the $\mathrm{H}^{+}$ and the negatively charged acceptor then follows compensation. The present calculations will confirm this suggestion, but will also show that the behavior of $\mathrm{H}$ is more complex, and depends upon the doping of the host material. Brief accounts of some of the major results of this work have been published elsewhere. ${ }^{17,18}$

Until recently, no experimental observations were available for isolated paramagnetic hydrogen centers. However, a large amount of experimental effort has been devoted to the study of muonium, a pseudoisotope of $\mathrm{H}$. The muon-spin-rotation technique allows the measurement of the hyperfine splitting of muonium, by studying the spin precession in a magnetic field. ${ }^{19}$ Two paramagnetic forms of $\mu$ have been observed: the so-called "normal" muonium $(\mathrm{Mu})$, with an isotropic hyperfine interaction, and "anomalous" muonium $\left(\mathrm{Mu}^{*}\right)$, with trigonal symmetry and a strong anisotropy of its hyperfine tensor. Normal muonium is usually associated with the tetrahedral interstitial site $(T)$. Recently, anomalous muonium was shown to be located at the bond center. ${ }^{20}$ This site was actually suggested by Cox and Symons, ${ }^{21}$ based on chemical arguments. It has to be noted that the muonium lifetime is only $2.2 \mu \mathrm{s}$, and its mass is $\frac{1}{9}$ that of H. Even though electronic properties do not depend on mass or lifetime, the observed behavior of muonium may differ from that of $\mathrm{H}$, and conclusions about muonium do not necessarily apply to $\mathrm{H}$. Nonetheless, we will see that certain of our results are in good agreement with the experimental observations on muonium. An extensive overview of the field of muonium in semiconductors has recently been compiled by Patterson. ${ }^{19}$

There has been one recent report of a paramagnetic hydrogen state, with indirect evidence that it would be associated with the bond center. Gordeev et al. ${ }^{22}$ observed by ESR a paramagnetic state due to $\mathrm{H}$ in $\mathrm{Si}$, called the $A A 9$ center. They also showed that the characteristics of $A A 9$ are similar to those of anomalous muonium $\left(\mathrm{Mu}^{*}\right)$. Since $\mathrm{Mu}^{*}$ is now known to be associated with the bond center $^{20}$ (a fact not appreciated in Ref. 22), this provides indirect evidence for the presence of a paramagnetic $\mathbf{H}$ state at the bond-center site.

Over the past ten years a number of theoretical studies were carried out that were aimed at determining the location and properties of $\mathrm{H}$ in Si. Many of these studies implicitly assumed that $\mathrm{H}$ would retain its atomic character in its interactions with bulk $\mathrm{Si}$, i.e., no strong binding to the crystalline network would occur, and $\mathrm{H}$ would favor interstitial locations where the interaction with the $\mathrm{Si}$ charge density would be minimal. This point of view led to the neglect of relaxation of the network in many of the earlier studies of the location of $\mathrm{H}$ in the $\mathrm{Si}$ crystal. It is now known, and will emerge very clearly from the present study, that relaxation of the host crystal around the $\mathrm{H}$ impurity is an essential feature of the interaction; most of the essential physics is missed when relaxation is not allowed. For instance, the global energy minimum for $\mathrm{H}$ in the positive and neutral charge states occurs at the bond-center position, i.e., midway between two $\mathrm{Si}$ atoms, provided these atoms are allowed to relaxed outward over a significant distance in order to accommodate the $\mathrm{H}$ atom. If no relaxation is allowed, $\mathrm{H}$ cannot insert into the bond.

An overview of the literature has been included in a recent review by Patterson. ${ }^{19}$ In the following we will not attempt a complete listing, but rather point out some relevant features and deficiencies of previous work. Among the first theoretical investigations were the extended-Hückel-theory cluster calculations of Singh et al. ${ }^{23}$ Empirical-pseudopotential Green's-function calculations were carried out by Rodriguez et al. ${ }^{24}$ Mainwood and Stoneham, ${ }^{25}$ using the semiempirical HartreeFock-based method of complete neglect of differential overlap (CNDO), addressed the possibility of different charge states for the $\mathrm{H}$. This issue was also addressed in the work of Johnson et al., ${ }^{5}$ where empirical tightbinding theory was used to derive the stable site for $\mathrm{H}$ in pure Si.

We also mention the empirical-pseudopotential supercell calculations of Pickett et al. ${ }^{26}$ even though they were carried out for $\mathrm{H}$ at the tetrahedral interstitial site in $\mathrm{Ge}$, not $\mathrm{Si}$. Their band structures showed a $\mathrm{H}$-induced deep donor state more than $6 \mathrm{eV}$ below the valence-band maximum. In contrast, recent calculations ${ }^{27}$ using $a b$ initio norm-conserving pseudopotentials have shown that $\mathbf{H}$ at $T$ in $\mathrm{Ge}$ induces a level just below the valence-band maximum, very similar to the situation in $\mathrm{Si}$. The erroneous result of Pickett et al. can be ascribed to lack of selfconsistency, and/or the use of empirical pseudopotentials. Starting from this result, it was argued that a spinpolarized treatment was necessary, which would introduce a shift in the defect level of up to $0.5 \mathrm{Ry}$, bringing it closer to the gap region. We will show in Sec. II E that this is incorrect, and that spin polarization has only a minor effect on the energy-level structure.

Katayama-Yoshida and Shindo ${ }^{28}$ actually carried out spin-density-functional calculations for $\mathrm{H}$ at the tetrahedral interstitial site in Si. They found a defect state in the upper part of the band gap. In our calculations (also including spin polarization) this state is close to and just below the top of the valence band. The result of Ref. 28 may be due to an insufficiently converged basis set.

A wide variety of cluster calculations have been applied to the problem. Besides the CNDO listed above, we mention the MNDO (modified neglect of diatomic overlap) method, used by Corbett et al., ${ }^{29}$ and minimalbasis-set Hartree-Fock calculations by Sahoo et al. ${ }^{30}$ More recent cluster calculations have included relaxation of the Si atoms: Estreicher ${ }^{31}$ has used the method of partial retention of diatomic differential overlap (PRDDO) and $a b$ initio minimal-basis-set Hartree-Fock calculations, and Deák and co-workers ${ }^{32}$ have applied the MINDO/3 (modified intermediate neglect of differential overlap) method. 
The results obtained from these cluster calculations display wide variations and inconsistencies, which illustrates the inadequacy of many of these methods to treat the problem at hand. The CNDO, MNDO, PRDDO, and MINDO/3 calculations are based on methods taken from quantum chemistry, which were developed to produce good results for molecules. Their application to solid-state problems, in which the semiconductor host is modeled by a cluster, is usually not justified. Very few cluster calculations test for convergence as a function of cluster size, or examine the effect of the termination of the cluster (usually with $\mathbf{H}$ atoms) and possible interactions with the defect states. Furthermore, the Hamiltonians used in those calculations contain parameters which are usually fitted to molecular properties. Certain aspects of local bonding may therefore be well reproduced, but there is no guarantee that the specific solid-state aspects of the interaction of the defect with a crystalline environment can be predicted. Systematic studies to investigate these problems in cluster calculations are very rare, and the few accounts that have been published are far from encouraging. For instance, Deák and Snyder ${ }^{33}$ concluded that MNDO, CNDO, and MINDO/3 all have serious difficulty in producing the band structure of the host lattice ( $\mathrm{Si}$ is found to be metallic in most of their cycliccluster calculations), and that calculated ground-state properties for defects may be subject to significant errors. One should therefore apply great caution in applying results from such calculations to the analysis of solid-state properties. The cluster calculations of Estreicher ${ }^{31}$ seem to have been tested most carefully for some of the potential problems mentioned above.

In contrast with most previous approaches, in this study we have used state-of-the-art theoretical methods which were developed with the explicit purpose of studying a wide variety of properties of solid-state materials. ${ }^{34}$ These techniques will be described in Sec. II. Section III contains our results for a single $\mathrm{H}$ atom in crystalline $\mathrm{Si}$; they are most clearly displayed in the form of totalenergy surfaces, which provide immediate insight into stable sites and low-energy paths for different charge states. We will also explore the stability of the different charge states in intrinsic, $p$-type and $n$-type material. Section IV deals with interactions between several $\mathbf{H}$ atoms, including molecule formation and mechanisms for defect formation. Section V contains a brief summary.

\section{METHODS}

The calculational procedure used in this work is based on density-functional theory in the local-density approximation $^{35}$ (LDA) and ab initio norm-conserving pseudopotentials. $^{36}$ The total energy is calculated using a momentum-space formalism: ${ }^{37}$ wave functions and potentials are expanded in plane-wave basis sets, and integrations over the first Brillouin zone are performed using the special-points algorithm. ${ }^{38} \mathrm{~A}$ thorough description of the theoretical approach can be found in Ref. 39 . Here, the properties of different charge states of $\mathrm{H}$ in $\mathrm{Si}$ are studied in a supercell geometry. ${ }^{40} \mathrm{We}$ carried out spin-polarized calculations for a number of representative configurations; the major conclusion is that the deviations from the spin-averaged calculations are small. Our results for total energies are most clearly displayed in the form of total-energy surfaces; we have used a novel technique to generate such surfaces, taking the full symmetry of the host crystal into account. We now proceed to describe and analyze each of these features in more detail.

\section{A. Pseudopotentials}

For Si we use a pseudopotential generated according to the Hamann-Schlüter-Chiang scheme, ${ }^{36}$ with cutoff radii of $0.99,1.49$, and 1.11 a.u., respectively, for $s, p$, and $d$ potentials. The $d$ potential is generated using an ionized configuration: $s p^{1} p^{0.75} d^{0.25}$. Test calculations carried out with this pseudopotential for $\mathrm{Si}$ in the diamond structure yield a theoretical lattice constant of $5.41 \AA$, and a bulk modulus of $0.94 \mathrm{Mbar}$ (to be compared with the experimental values of $5.43 \AA$ and 0.99 Mbar). At an energy cutoff of $(6 ; 12) \mathrm{Ry}$, at which most of our calculations for lattice relaxations are carried out, the theoretical lattice constant is $5.42 \AA$. [The notation $\left(E_{1} ; E_{2}\right) \mathrm{Ry}$ means that plane waves with kinetic energy up to $E_{2}$ Ry are included in the expansions of wave functions and potentials; waves with kinetic energy up to $E_{1} \mathrm{Ry}$ are included in an exact diagonalization of the Hamiltonian matrix, while those with kinetic energy between $E_{1}$ and $E_{2}$ Ry are included in second-order Löwdin perturbation theory. ${ }^{41} \mathrm{We}$ always choose $E_{2}=2 E_{1}$.] These results indicate the reliability of the $\mathrm{Si}$ pseudopotential for structural studies.

The band structure produced by this pseudopotential is in satisfactory agreement with experiment, except for the well-documented problem of local-density-functional theory that the band gap is too small. We calculate a conduction-band minimum along the $\Gamma-X$ direction, at about 0.8 times the distance to the $X$ point, at $0.48 \mathrm{eV}$ above the top of the valence band. The implications for our defect calculations will be discussed later.

For $\mathrm{H}$ we have simply used the Coulomb potential. Test calculations have shown that no gain in convergence properties is obtained by using a pseudopotential, and that the $1 / r$ divergence of the Coulomb potential near the core presents no difficulties.

\section{B. Plane-wave basis set}

We have performed extensive tests to establish the convergence as a function of the plane-wave basis set which is used for expanding wave functions and potentials. Typical plots showing convergence of total-energy differences as a function of energy cutoff are shown in Fig. 1. The ordinate shows the energy difference between two reference configurations in an eight-atom cell. (The eight-atom cell size is generally two small for extracting meaningful results, but is adequate for a study of the plane-wave convergence.) Figure $1(a)$ is for the energy difference between $\mathrm{H}^{-}$at the $T$ site and $\mathrm{H}^{-}$at the hexagonal interstitial site $(H)$. Figure $1(b)$ is for the energy difference between $\mathrm{H}^{-}$at the $T$ site and $\mathrm{H}^{-}$at the bondcenter site (including only first-neighbor relaxation). The negative charge state was chosen to avoid problems with 
level occupations. Indeed, the $\mathbf{H}$ atom induces a level near the band gap in the band structure, which has to be left unoccupied (half-occupied) in the positive (neutral) charge state. The occupation for the negative charge state is most easily accomplished, since it only requires filling up all levels with two electrons. Charge states are discussed in more detail in Sec. II F. The abscissa of both plots in Fig. 1 is the energy cutoff $E$ used in the planewave expansions: plane waves with kinetic energy up to $E / 2$ are included in an exact diagonalization of the Hamiltonian matrix, while those with kinetic energy between $E / 2$ and $E$ are included in second-order Löwdin perturbation theory. ${ }^{41}$

In Fig. 1(a) the energy difference is converged (within $0.01 \mathrm{eV}$ of its final value) at a cutoff of $(9 ; 18) \mathrm{Ry}$. Figure 1(b), for the bond center, shows that at $(9 ; 18) \mathrm{Ry}$ the energy difference is within $0.15 \mathrm{eV}$ of its final value, which is only reached above $(18 ; 36)$ [note the different scale of panels 1 (a) and 1(b)]. We thus see that the convergence properties depend on the position of the $\mathrm{H}$ atom. In fact, as we will see later, one can distinguish two regions in the $\mathrm{Si}$ crystal in which the $\mathbf{H}$ impurity shows distinctly different behavior. The first region is that of high electron density, including the bond-center site $(B)$, the sites $C$ and $C^{\prime}$ (at the center of a rhombus formed by three adjacent $\mathrm{Si}$ and the nearest $T$ ), etc. The location of these sites is illustrated in Fig. 2. The second region consists of
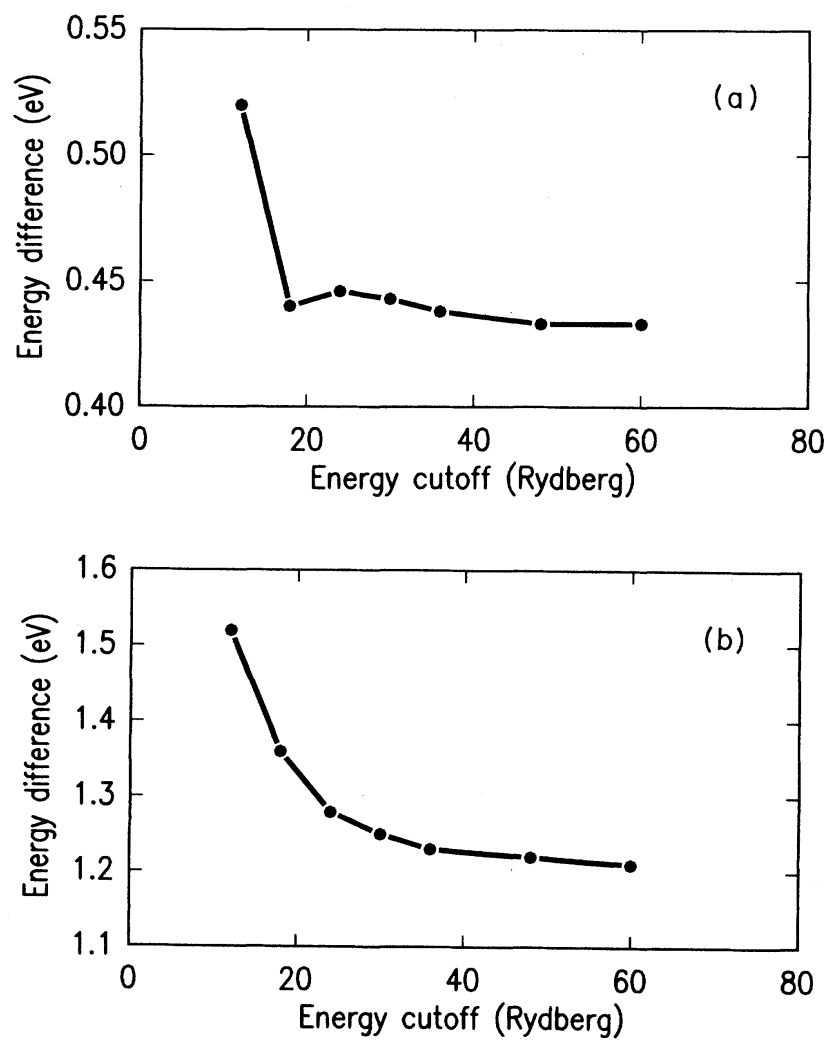

FIG. 1. Convergence of total-energy differences between reference configurations in an eight-atom cell, as a function of energy cutoff. (a) is for the energy difference between $\mathrm{H}^{-}$at $T$ and at $H$. (b) is for the energy difference between $\mathrm{H}^{-}$at $T$ and at $B$.

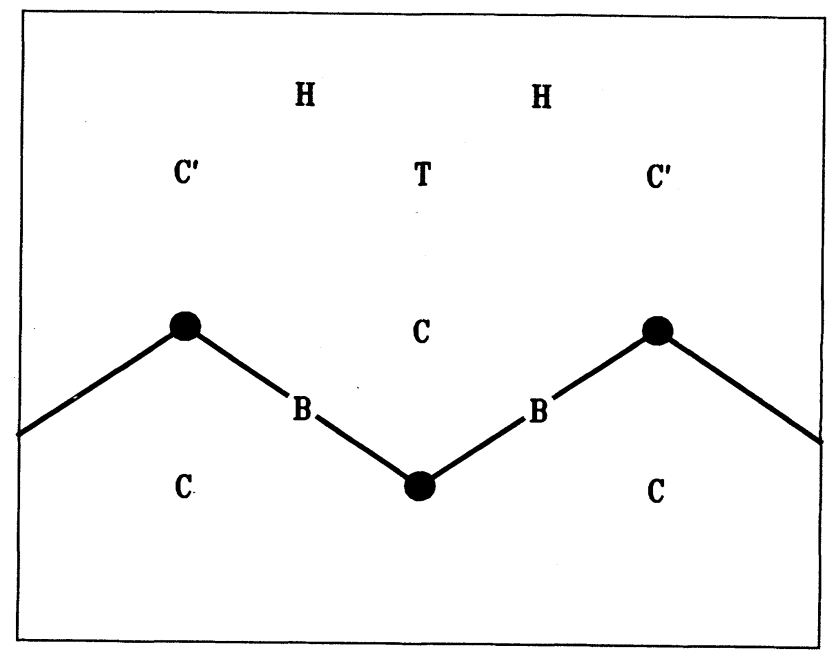

FIG. 2. Schematic illustration of the (110) plane through the atoms in the Si crystal, with labels for relevant high-symmetry positions. $T$ is the tetrahedral interstitial site, $H$ is the hexagonal interstitial site, $B$ the bond center, and $C$ (and $C^{\prime}$ ) is at the center of a rhombus formed by three adjacent $\mathrm{Si}$ atoms and the nearest $T$ site.

the low-electron-density "channels" and includes the high-symmetry tetrahedral $(T)$ and hexagonal $(H)$ interstitial sites. Energy differences between $H$ positions located within the same region generally converge quite fast [cf. Fig. 1(a)], while those between positions in different regions [cf. $B$ and $T$, in Fig. 1(b)] are slower in convergence. Reference configuration (b) was chosen for this test because it presents the extreme "worst case" in terms of convergence properties; energy differences between other configurations have consistently been found to converge faster than presented in Fig. 1(b). We have also carried out test calculations in 16- and 32-atom supercells up to $(12 ; 24) \mathrm{Ry}$ that confirm that the behavior as a function of cutoff is the same for all cell sizes.

We have found that inclusion of plane waves with kinetic energy up to $(6 ; 12) \mathrm{Ry}$ (i.e., waves up to $6 \mathrm{Ry}$ included in exact diagonalization, between 6 and $12 \mathrm{Ry}$ in second-order perturbation theory) is sufficient for obtaining the general features of the energy surfaces. Since some of the configurations (e.g., the $B$ site) are particularly sensitive to the energy cutoff, all of the calculations necessary to derive energy differences for those sites were also carried out at the higher cutoff of $(9 ; 18) \mathrm{Ry}$. This cutoff corresponds to a basis set of $\sim 5500$ plane waves in the 32 -atom cells. The values for energy differences and barriers that will be quoted all result from these highcutoff calculations. This may lead to small quantitative differences with values that were quoted in earlier brief accounts of the present work. ${ }^{17,18}$

Finally, we have also examined the reliability of Löwdin second-order perturbation theory ${ }^{41}$ for deriving total-energy differences in this system, by comparing results obtained at $(E / 2 ; E) \mathrm{Ry}$ with those obtained at $(E ; E)$, i.e., without any perturbation theory, for various values of the cutoff $E$. We found that the deviations were always smaller than $0.1 \mathrm{eV}$ [e.g., only $0.07 \mathrm{eV}$ at $(6 ; 12)$ 
Ry], and decreased rapidly to zero when the cutoff was increased [being smaller than $0.01 \mathrm{eV}$ at $(15 ; 30) \mathrm{Ry}$ ], showing that the values converge to the same limit at infinite cutoff.

\section{Supercells}

To study the atomic and electronic structure of an impurity in the crystalline environment, while still preserving the translational symmetry of the system required for our theoretical formalism, we artificially introduce periodicity by constructing a supercell in which the impurity is surrounded by a sufficiently large number of $\mathrm{Si}$ atoms. We typically use supercells containing $32 \mathrm{Si}$ atoms, such that the distance between neighboring defects if $9.4 \AA$. The convergence as a function of supercell size was tested by performing calculations on supercells containing 8,16 , and $32 \mathrm{Si}$ atoms. For energy differences between $\mathbf{H}$ positions in the same (high- or low-electrondensity) region, the 16-atom cell was found to suffice; energy differences between different locations for the same charge state were converged to within $0.1 \mathrm{eV}$, and differences between different charge states to within 0.2 eV. For energy differences between $\mathbf{H}$ positions in highversus low-density regions, however, the error bar in a 16 -atom cell is larger. Compared with a 32 -atom cell, deviations of up to $0.4 \mathrm{eV}$ may occur in the energy differences. These deviations can be attributed to the larger extent of the defect wave functions, as observed in plots of the charge density (see Sec. III B), which causes more significant interactions between neighboring 16atom supercells for $\mathrm{H}$ at the bond center.

The 32-atom cells also facilitate the extraction of band positions for an isolated defect, since the dispersion of this level (caused by interactions with defects in neighboring supercells) is less than $0.5 \mathrm{eV}$ in this case. The position of the level that would correspond to an isolated defect (i.e., without dispersion) was determined by taking a weighted average over the band positions at the special points. It changed by less than $0.1 \mathrm{eV}$ when the cell size was increased from 16 to 32 atoms.

Dispersion of the defect levels due to interactions between neighboring supercells places an error bar on the derived position for any defect level. A more important source of uncertainty, however, is due to the intrinsic deficiencies of the local-density approximation (LDA), particularly the fact that the LDA predicts conduction bands and hence conduction-band-derived energy levels to be too low. We will therefore refrain from quoting specific results for positions of energy levels in the band gap. We note, however, that a qualitative distinction between various positions of the $\mathrm{H}$-induced level can still be made. We also note that, while the absolute position of the defect level is uncertain, its relative motion induced by displacements of the impurity or by changes in the charge state is quite reliable. These observations will allow us to derive conclusions about the deep levels induced by hydrogen, as described later in the paper. Only in the section where we discuss the relative stability of various charge states as a function of the Fermi-level position will we be confronted with the limitations of the
LDA.

For each position of the impurity, we need to let the neighboring $\mathrm{Si}$ atoms relax to find the lowest-energy configuration. Relaxation of two shells of Si atoms surrounding the $\mathrm{H}$ impurity is included in the full calculations. The need to relax two shells of $\mathrm{Si}$ atoms was another reason to carry out the calculations in 32-atom supercells, since 16-atom cells are too small to include anything but first-neighbor relaxations in a meaningful way. Second-neighbor relaxations can lower the energy by several tenths of an $\mathrm{eV}$, e.g., for $\mathrm{H}$ at the bond center. Relaxation of further shells causes less than $0.1 \mathrm{eV}$ change in the total energy, as was checked with a Keating model. ${ }^{42}$

\section{Special points}

Integrations over the first Brillouin zone are performed using the special-points scheme. ${ }^{38}$ In the 32 -atom cells, two special points in the irreducible part of the zone are used for trigonal symmetry situations (e.g., $\mathrm{H}$ on the extension of a Si-Si bond), and equivalent larger sets for lower-symmetry configurations. We test the convergence as a function of the special-point sample as follows: If we increase the parameter $q$ in the Monkhorst-Pack ${ }^{38}$ scheme from 2 to 4 , the number of special points generated in the irreducible part of the Brillouin zone increases from two to seven for the $T$ site, and from two to twelve for the $B$ site. Even though the absolute value of the total energy changes significantly when the larger $k$-point set is used, all energy differences between different sites change by less than $0.05 \mathrm{eV}$.

\section{E. Spin polarization}

In this work we report local-density-functional results for total energies and defect levels; spin polarization, ${ }^{28}$ which affects only the neutral charge state (with an unpaired electron), was not included. We established the validity of this approach by carrying out self-consistent spin-density-functional calculations, which are much more time consuming, at selected sites. For the bondcenter position the inclusion of spin polarization has very minor effects: the total energy goes down by less than $0.02 \mathrm{eV}$, and the defect level is split by only $0.04 \mathrm{eV}$.

The deviation from the spin-averaged results is expected to be largest for $\mathrm{H}$ at the $T$ site, where the crystal charge density reaches its lowest value so that the impurity is most "free-atom"-like. (Note that the $T$ is not a stable site for $\mathrm{H}^{0}$ in $\mathrm{Si}$, as we will see in the next section.) It is worthwhile to point out here, for the purposes of the present study, namely the derivation of total energies, what the effect is of neglecting spin polarization in the LDA calculation for the free $\mathbf{H}$ atom. The total energy deviates from the spin-polarized value by $\sim 0.9 \mathrm{eV}$. The error can be associated with the absence of exchange splitting, which would lower the occupied level. In the solid, such exchange splittings are known to be substantially reduced from the free-atom values; this was observed in calculations for transition-metal impurities. ${ }^{43}$ These qualitative arguments were confirmed by the full spin-polarized calculations. For $\mathrm{H}^{0}$ at the $T$ site, we 
found that inclusion of spin polarization lowered the total energy only by $0.1 \mathrm{eV}$. The defect level was split into a spin-up and a spin-down level, which were separated by $0.37 \mathrm{eV}$. These results are consistent with spin-polarized linearized muffin-tin orbital (LMTO) Green's-function calculations for $\mathrm{H}$ in $\mathrm{Si}^{44}$

The overall conclusion is that the effects of spin polarization on the total energy are very small. They are therefore not included in the calculations that lead to the total-energy surfaces presented in the next section. We will, however, show contour plots of spin densities, which provide valuable information about the electronic structure of the impurity at different sites.

\section{F. Charge states}

The calculation of charge states requires careful treatment, since the LDA pseudopotential expressions for the total energy are all derived assuming charge neutrality in the unit cell. ${ }^{37,39}$ Such neutrality is indeed necessary to avoid divergence of the long-range Coulomb terms. Taken individually, the $\mathbf{G}=\mathbf{0}$ terms of the electron-ion, electron-electron (Hartree), and ion-ion interactions are infinite. A finite result is obtained, however, by appropriate combination of terms, leading to two well-defined and finite contributions: (1) the Ewald energy, which is the energy of a periodic array of positive point ions in a uniform neutralizing (negative) background, and (2) the socalled $\alpha Z$ term, ${ }^{37}$ which represents the Fourier component for $\mathbf{G}=\mathbf{0}$ of the electron-(pseudo)ion interaction.

Our approach for performing the calculations on a charged system is as follows: We define the occupation of the electronic energy levels to represent the system that we want to study (i.e., positively or negatively charged, by taking out an electron, or putting in an extra electron with respect to the neutral system). The charge density is then calculated from the wave functions of the occupied states, and all summations in the total-energy terms, as well as the generation of a new potential in the selfconsistent process, are carried out with this charge density. However, the $\mathbf{G}=\mathbf{0}$ terms (i.e., the Ewald and $\alpha \boldsymbol{Z}$ terms) are always calculated for the neutral system (the charge being determined by the ionic charges in the supercell). Neutrality is essential here, because a nonneutral system would surely lead to diverging terms. It is also the appropriate approach to the physics problem, for the following reasons.

Since charge neutrality is a fundamental requirement, all calculations should really be set up with a number of electrons that exactly equals the number of positive charges in the unit cell. Since the latter is determined by the structure, use of the Ewald and $\alpha Z$ terms for the neutral system is appropriate. The neutrality condition leads, strictly speaking, to the requirement of the presence of an additional charge that would compensate the extra charge in our system. Specifically, if we put an extra electron on a defect, then we should have a hole (compensating positive charge, or absence of an electron) present in the same unit cell-and very far removed from the extra electron, so as not to lead to spurious interaction terms. This setup is generally impractical, since it leads to a requirement of very large supercells, and makes separation of the terms in the total energy that are related to the defect (and not to the compensating charge) very difficult. Nevertheless, it was used by Vanderbilt and Joannopoulos ${ }^{45}$ in a study of defects in Se, in conjunction with an elaborate scheme for specifying level occupations.

Since the compensating charge is not supposed to interact with the charge on the defect, and basically only serves to maintain charge neutrality for the calculation of $\mathbf{G}=\mathbf{0}$ terms - something which we impose anyway-we can take the shortcut of leaving it out of the calculation altogether. By doing this, we are neglecting a Madelung-type term which would describe the interaction between (screened) positive and negative charges, and which would vanish in the limit of an infinite supercell. This approach will therefore be justified if the results are shown to be converged as a function of supercell size. In that case, the supercell is large enough to avoid spurious interactions between charged defects in neighboring cells. A test as a function of supercell size is therefore essential, and has been carried out in this study with satisfactory results.

As a final check on the procedure, we have examined one test case in which the "strict" application of charge neutrality was obeyed, by having two oppositely charged defects present in the supercell. Our calculations on individual defects in a 32-atom cell (to be described in more detail in the next section) established that at the bondcenter site $\mathbf{H}$ is most stable in the positive charge state, while at the tetrahedral interstitial site the negative charge state is favored. We also obtained values for the total energies for each of these configurations. We then proceed to construct a 32-atom supercell in which both defects are present at the same time: one impurity at a bond center, $B$ (with appropriate relaxation of the surrounding lattice), the other at the tetrahedral interstitial site $(T)$. The minimum-energy electronic structure for this arrangement should put the $\mathrm{H}$ at $B$ in the positive charge state, and the $\mathrm{H}$ at $T$ in the negative charge state. This is indeed what we find by analyzing the charge density. This supercell is now overall neutral (since it contains one positive and one negative defect), and therefore the calculation strictly follows the treatment of $\mathbf{G}=\mathbf{0}$ terms, as discussed above. Such an arrangement of defects therefore follows the scheme proposed by Vanderbilt $^{45}$ for performing calculations for charged states. We want to check whether the total energy obtained from this calculation is equal to the sum of the total energies obtained from separate calculations for the individual defects. Spurious interactions between the positive and negative charges within the unit cell (and with neighboring cells) may be present, of course; assuming, however, that the defects are sufficiently well separated so that the only interaction would be through a screened Coulomb interaction, the resulting changes in the total energy would be quite small. That allows us to obtain a value for the total energy for a pair of (to a good approximation) noninteracting defects. This total energy turns out to be the same (within $0.1 \mathrm{eV}$ ) as the sum of total energies obtained from calculations for individual defects, in which the prescription outlined above was followed. This agree- 
ment confirms the validity of our prescription for charge states.

\section{G. Energy surfaces}

To study the behavior of an impurity (in a particular charge state) in a semiconductor, one needs to know the total energy of many different configurations, in which the impurity is located at different sites in the host crystal. For each position of the impurity, the surrounding atoms should be completely relaxed. The resulting energy values as a function of the coordinates of the impurity define an energy surface: $E=E\left(\mathbf{R}_{\text {imp }}\right)$. Notice that this function does not depend on the coordinates of the host atoms; that is because for each position $\mathbf{R}_{\text {imp }}$ an energy minimization procedure has been performed (i.e., relaxation) that determines what the coordinates of the host atoms are. Once the function is known, it immediately provides information about stable sites, migration paths, and energy barriers along these paths.

A function such as $E=E\left(\mathbf{R}_{\mathrm{imp}}\right)$ that depends on three dimensions is difficult to visualize. Symmetries of this object can play an important role in simplifying both the calculational task and the conceptual understanding. The crucial point here is the realization that the energy surface possesses the full symmetry of the crystal. To make effective use of this symmetry, an analytic description of the surface is essential. We achieve this through expansion in a basis set with the appropriate symmetry. A natural choice for a basis set with the full symmetry of the lattice is a set of symmetrized plane waves. Such a description is used, for instance, in self-consistent planewave basis-set calculations to represent charge densities; experience indicates that relatively few coefficients suffice to adequately describe the overall features of the function. A progressively better description can be obtained by including more symmetrized plane waves.

In order to test the representation, we worked with a large data base of energy values (more than 16 locations of $\mathrm{H}$ in the lattice). For each test we selected a particular subset of these, containing $m$ values, and used this set to determine the expansion coefficients when $n$ basis functions (symmetrized plane waves) were included in the expansion of the energy surface. With $m \geq n$, a leastsquares-fitting procedure was used. We found that a minimum of six basis functions is required to represent the features of the surface; the quality of the representation could be judged by taking the predicted energy value at a data point that was not included in the subset of $m$ points, and comparing it with the value that was independently calculated from first principles. Increasing the number of basis functions from eight to ten led to energy changes in the relevant areas of the surface of less than $0.05 \mathrm{eV}$. (Near the atoms, the surface rises very rapidly; relative variations with the number of basis functions may be larger in these regions, but are of no consequence for the physical behavior). We conclude that eight to ten calculated data points suffice to determine the expansion coefficients.

While it is impossible to pictorially represent the energy surface as a function of all three dimensions, our choice of data points and our fitting procedure assure that we take the full three-dimensional character into account. For visualization, we restrict the coordinates of the $\mathrm{H}$ impurity to a particular plane [e.g., the (110) plane through the atoms]. The energy surface can then be displayed as a contour plot (the curves presenting lines of constant energy), or as a perspective plot of the energy (along the vertical axis) as a function of the coordinates in the plane. Both types of plot will be used here. Note that the Si relaxations for each position of the impurity atom are different but are not displayed in these figures.

In Figs. 3-5 we show contour plots and perspective plots for different charge states of $\mathbf{H}$ in $\mathbf{S i}$, with the impurity coordinates restricted to the (110) plane through the atoms. In all plots the (arbitrary) zero of energy is at the $T$ site. The contour plots are self-explanatory; highsymmetry sites (cf. Fig. 2) have been included for easy inspection. The perspective plots have been color-coded to allow straightforward identification of the relevant regions. Red regions present the lowest-energy values, blue is intermediate, and green is for the highest energies. The plots should be interpreted as a perspective view of a landscape, in which the low-lying regions ("valleys") represent the most favorable positions for the impurity. The plateaus around the perfect-crystal $\mathrm{Si}$ atomic sites are not real: when the $\mathrm{H}$ atom approaches any $\mathrm{Si}$ atom too closely, the energy rises rapidly; this gives rise to a very steep "mountain" in the surface, which would obscure everything behind it in a perspective plot. We have therefore cut off these mountains at a value listed as the upper limit of the green regions in the plots. A quantitative discussion of these plots will be given in the next section.

\section{RESULTS FOR A SINGLE HYDROGEN IMPURITY IN CRYSTALLINE SILICON}

The energy surfaces for $\mathbf{H}$ in the positive, neutral, and negative charge states, as depicted in Figs. 3-5, exhibit a number of common features. In all three charge states there are two distinct regions in which the $\mathrm{H}$ atoms exhibit significantly different behavior. First, there is the region of high electron density, which includes the $B$ (bond-center) site and the $C$ site (at the center of a rhombus formed by three adjacent $\mathrm{Si}$ and the nearest $T$ ). In this region the nearby $\mathrm{Si}$ atoms relax strongly. For example, when the $\mathrm{H}$ atom is placed at the bond-center site, the adjacent $\mathrm{Si}$ atoms relax out by $0.4 \AA$ for a net gain in energy of more than $4 \mathrm{eV}$. If no relaxation were included, the red low-energy region in Fig. 3(b) would completely disappear. Figure 6 shows the energy surface calculated for a rigid Si lattice; the bond-center and other positions in the high-density region are indeed high in energy now. Coming back to the case in which relaxation is included, we find that in the high-density region a $\mathrm{H}$ induced defect level occurs in the upper part of the energy gap; it is identified as a state formed out of an antibonding combination of $\mathrm{Si}$ orbitals. The second region consists of the low-electron-density "channels" and includes the high-symmetry tetrahedral $(T)$ and hexagonal $(H)$ interstitial sites. Here, the Si atoms in the vicinity of 
$\mathrm{H}$ relax very little if at all. Furthermore, a H-related level now occurs just below the top of the valence bands. The precise position of the defect levels changes only by $\sim 0.1 \mathrm{eV}$ as a function of charge state. We now discuss the various charge states and their relative stability in more detail.

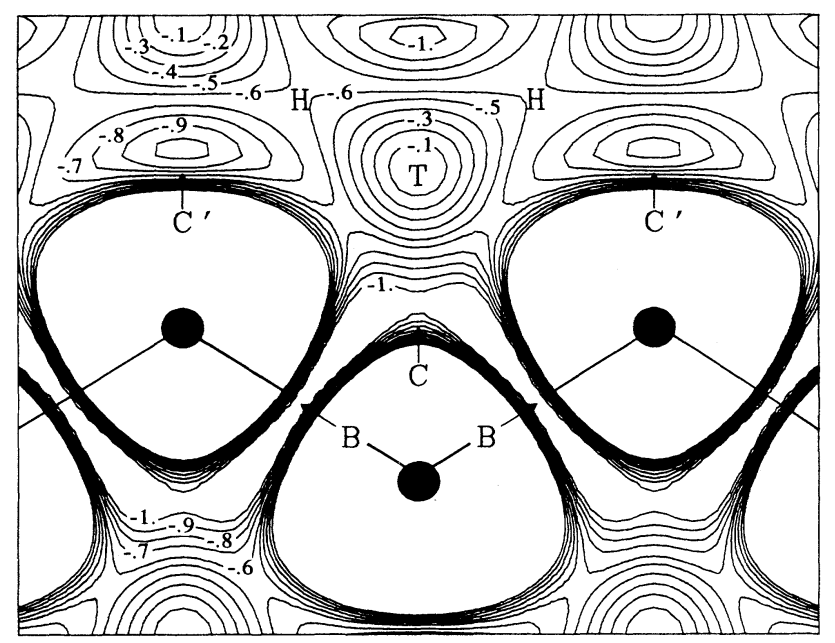

(a)

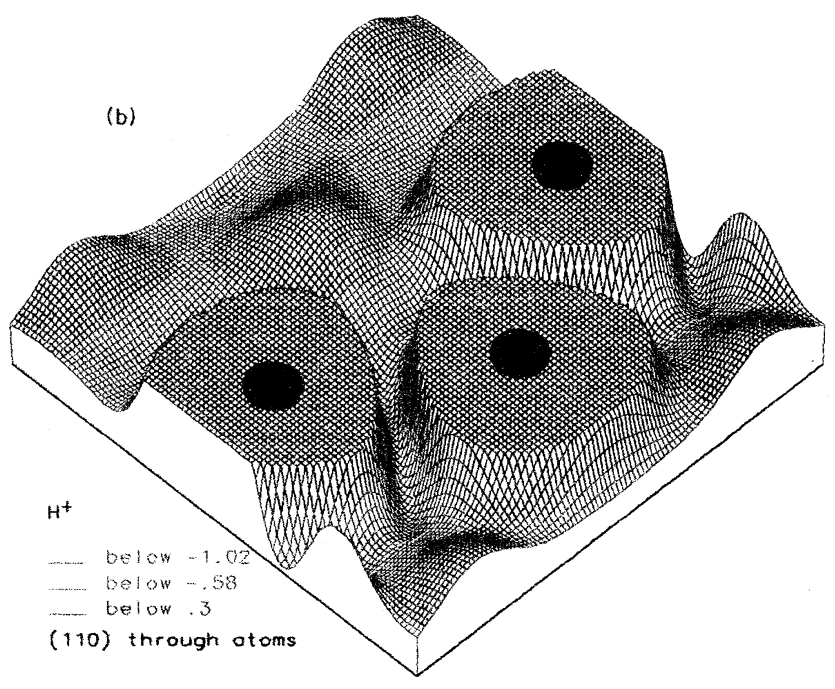

FIG. 3. (a) Contour plot and (b) perspective plot of the energy surface for $\mathrm{H}^{+}$in a (110) plane through the Si atoms. The zero of energy is arbitrarily chosen at $T$. The black dots represent $\mathrm{Si}$ atoms at their unrelaxed positions; the relaxations (which are different for different $\mathrm{H}$ positions) are not shown but are taken into account in the total-energy calculations. In (a) the contour interval is $0.1 \mathrm{eV}$. The color coding of the perspective plot in (b) is indicated in the figure: the energy values below $-1.02 \mathrm{eV}$ are shown in red; between -1.02 and $-0.58 \mathrm{eV}$ in blue; and between -0.58 and $0.3 \mathrm{eV}$ in green. The surface is cut off at an energy value of $0.3 \mathrm{eV}$.

\section{A. Positive charge state}

Figure 3 shows the energy surface in the (110) plane for a positively charged $\mathbf{H}\left(\mathbf{H}^{+}\right)$. The global minimum is at the bond center $(B)$ site, symmetrically located between two $\mathrm{Si}$ atoms. In contrast, the energy of $\mathrm{H}^{+}$in the lowdensity region is more than $0.5 \mathrm{eV}$ higher (the bond center is $1.2 \mathrm{eV}$ lower in energy than the $T$ site). Of course, the state $\mathrm{H}^{+}$in the low-density region actually does not occur, because the H-related level which must be kept empty lies inside the valence bands. Note that the positive charge state does not imply that $\mathrm{H}$ occurs as a bare proton; at the bond center, the missing charge is actually taken from the region near the $\mathrm{Si}$ atoms, corresponding to the state occurring in the band gap. In a simplified picture of combination of orbitals on the $\mathrm{H}$ and

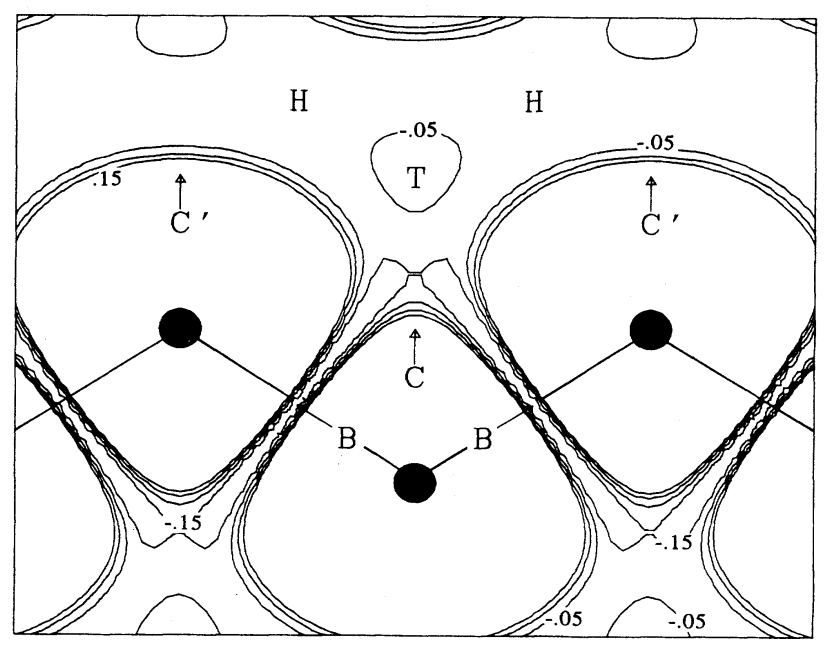

(a)

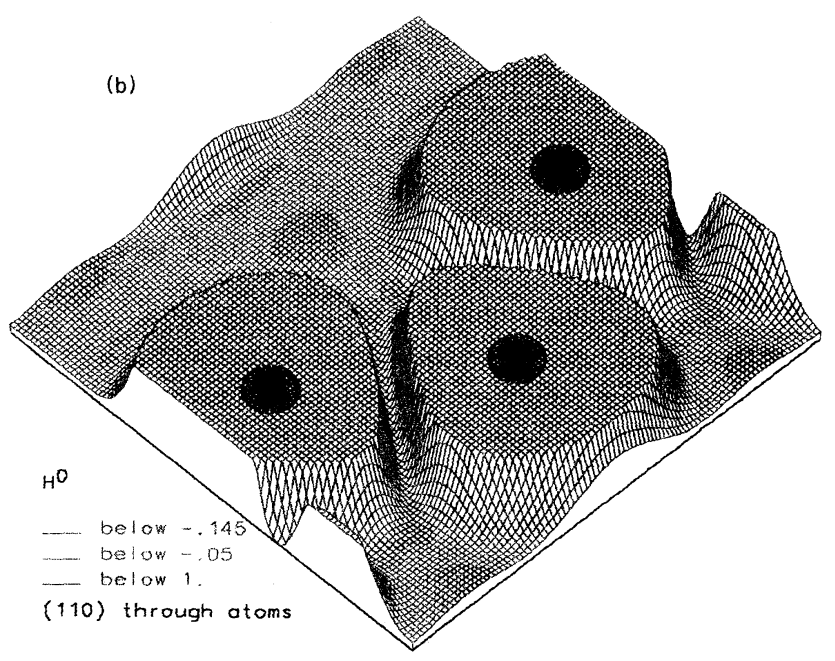

FIG. 4. (a) Contour plot and (b) perspective plot of the energy surface for $\mathrm{H}^{0}$ in a (110) plane through the $\mathrm{Si}$ atoms. See caption of Fig. 3. 
the neighboring $\mathrm{Si}$ atoms, this state can be considered to be formed out of an antibonding combination of Si orbitals; viewed as a state of the defect complex, it is effectively nonbonding in character, since it has a node through the $\mathbf{H}$ atom. For $\mathbf{H}$ at the bond center, our use of the notation $\mathrm{H}^{+}$therefore implies that the actual defect is a complex formed by the $\mathrm{H}$ and the surrounding $\mathrm{Si}$, the electron being removed from an antibonding combination of $\mathrm{Si}$ orbitals rather than from the $\mathrm{H}$ itself.

A migration path in the (110) plane can be traced between the bond-center positions; the barrier along this path is $\sim 0.2 \mathrm{eV}$ high. This path can clearly be seen as the red region winding its way around the $\mathrm{Si}$ atoms in

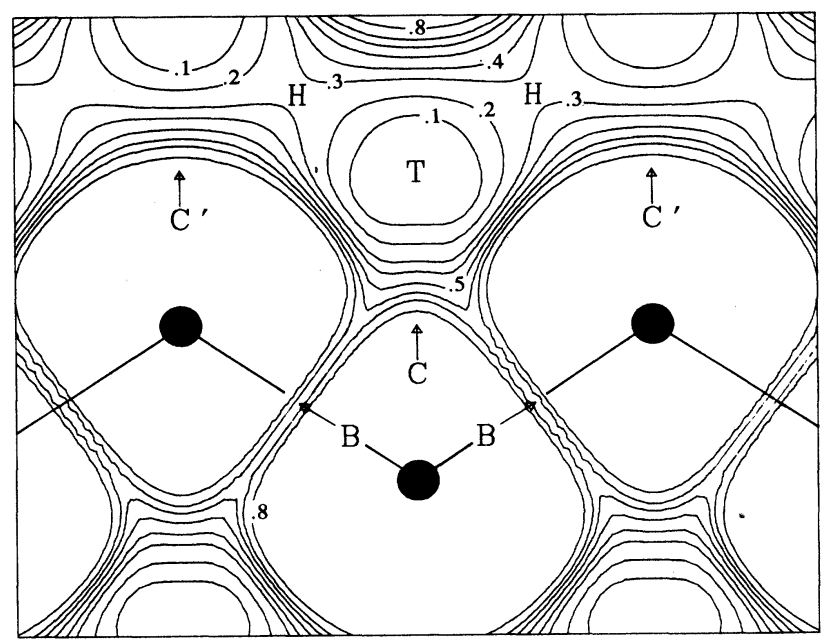

(a)

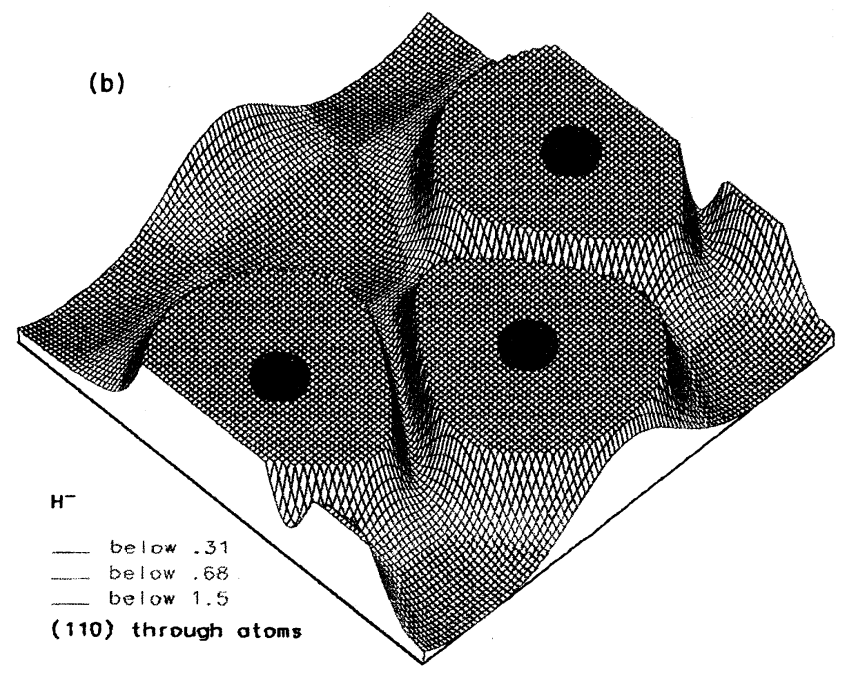

FIG. 5. (a) Contour plot and (b) perspective plot of the energy surface for $\mathrm{H}^{-}$in a (110) plane through the Si atoms. See caption of Fig. 3.

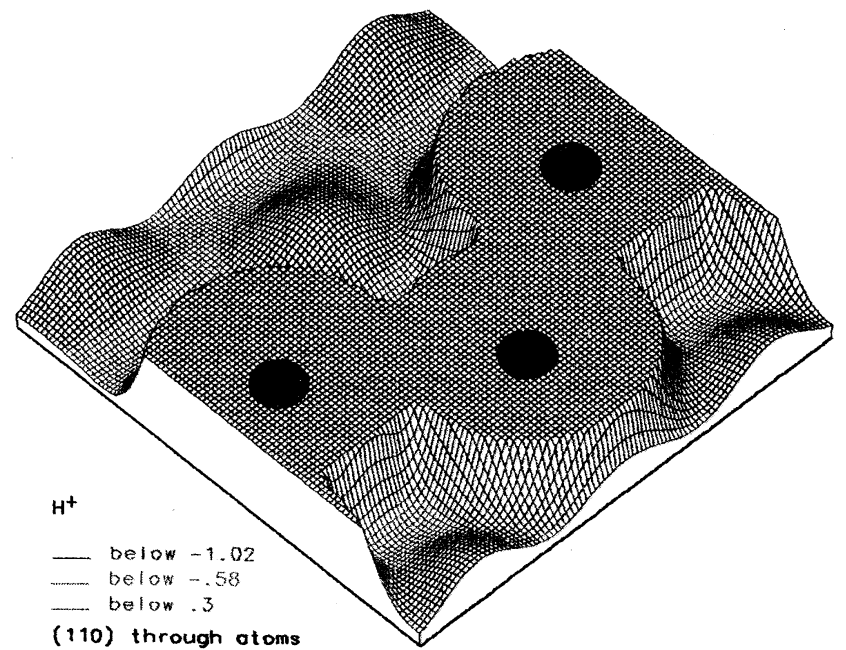

FIG. 6. Perspective plot of the energy surface for $\mathbf{H}^{+}$in a (110) plane through the $\mathrm{Si}$ atoms. To generate the values for this plot (unlike all others) the Si atoms were kept fixed in their rigid lattice positions. Comparing with Fig. 3, we see that the low-energy regions have disappeared.

Fig. 3(b). The saddle point occurs very close to the point indicated with $C$ in the contour plot; the points $C^{\prime}$ are symmetry-related points along equivalent paths perpendicular to the plane of the figure. At the saddle point in the (110) plane, $\mathbf{H}$ is located $1.25 \AA$ away from the $T$ site. The Si atom below it, on the line through $T$ and the saddle point, relaxes down by $0.16 \AA$ to make the Si-H distance equal to $1.63 \AA$. Since we cannot show the energy surface as a function of all three dimensions, the (110) plane and the indicated migration path should only be considered as a representative example. We have also studied the behavior in various other planes. Figure 7 shows the energy surface in a (110) plane parallel to the plane in Fig. 3 and lying halfway between equivalent planes through the atoms. In particular, we are interested in the behavior around the $M$ site, which is midway between two $C$ sites [only one of which lies in the (110) plane]. Corbett et al. ${ }^{29}$ proposed this site as the minimum-energy location for neutral $\mathbf{H}$ in $\mathrm{Si}$. In our energy surfaces for $\mathbf{H}^{+}$, we find it to be at approximately the same energy as the bond center $B$, with no barrier between the two. Migration along a path involving the $M$ sites still involves a barrier of $\sim 0.2 \mathrm{eV}$. As can be seen in Fig. 7 , the $M$ point also lies on a line perpendicular to the $\mathrm{Si}-\mathrm{Si}$ bond, connecting the bond center with the neighboring hexagonal interstitial site; all points between $B$ and $M$ on this line have approximately the same energy. For these "buckled" configurations, the Si-H distance remains almost constant (equal to $1.6 \AA$ ), due to appropriate relaxation of the $\mathrm{Si}$ atoms. In all cases, the $\mathrm{H}$ prefers to be symmetrically located with respect to two $\mathrm{Si}$ atoms.

Figure 8, finally, shows a cross section which contains the $B, M$, and $H$ sites, but also goes through the atoms. The flatness of the surface along the line from $B$ to $M$ is once again evident. It can also be observed that the ener- 
gy surface rises steeply along the bond direction ([111]).

For $\mathbf{H}$ at the bond center itself, in the positive charge state, the neighboring $\mathrm{Si}$ atoms move out over $0.41 \AA$, to make the Si-H distance equal to $1.59 \AA$. This distance is slightly larger than the $\mathrm{Si}-\mathrm{H}$ bond length in molecules such as $\mathrm{SiH}_{4}$, where it is $1.48 \AA$. This is understandable since $\mathrm{H}$ at the bond center is bonded to two $\mathrm{Si}$ atoms, forming a three-center bond. The second neighbors move by $0.07 \AA$; the distance between first and second neighbors is equal to $2.31 \AA$.

These motions of the $\mathrm{Si}$ atoms are quite large, and must involve a significant energy cost. To estimate this

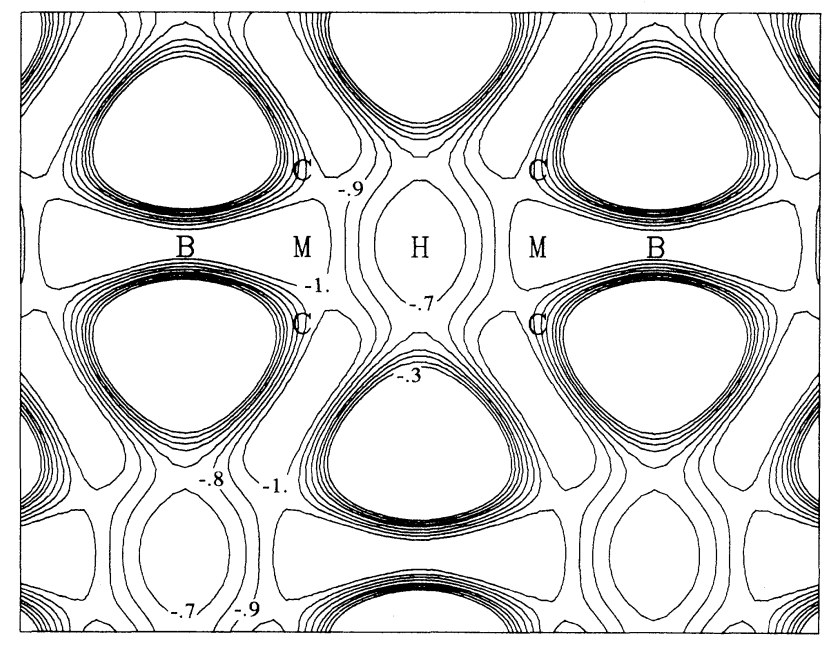

(a)

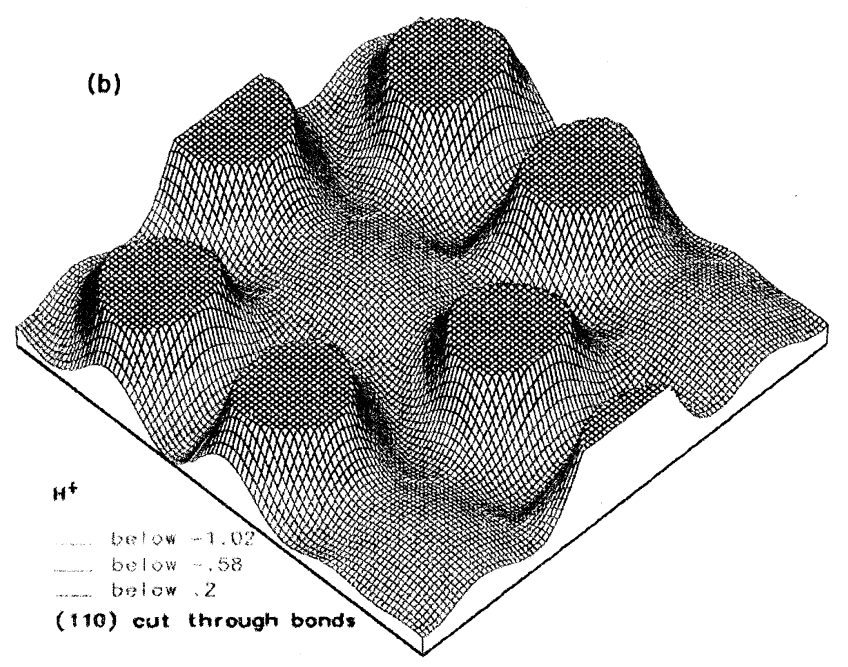

FIG. 7. (a) Contour plot and (b) perspective plot of the energy surface for $\mathrm{H}^{+}$in a (110) plane through the sites $B, C, H$, and $M$. This plane is parallel to the plane of Fig. 3, and midway between equivalent planes through the atoms. The $M$ point is located midway between a bond center and the nearest hexagonal interstitial site. The zero of energy is arbitrarily chosen at $T$ (not in the plot). See caption of Fig. 3. raise in energy due to strain, we have performed a calculation of the $\mathrm{Si}$ atoms in the positions described above, but in the absence of the $\mathrm{H}$ impurity. The total energy is $1.55 \mathrm{eV}$ higher than for the lattice in equilibrium. This means that the energy gained due to bonding between $\mathbf{H}$ and $\mathrm{Si}$ must be greater than $1.55 \mathrm{eV}$, in order for the bond-center configuration to be stable. This "cost of relaxation" can also be interpreted in the following fashion: If a situation could be created in which outward motion of the $\mathrm{Si}$ atoms would levy no energy cost, the bondcenter configuration for $\mathrm{H}^{+}$would be more stable by 1.55 $\mathrm{eV}$, compared to the situation in crystalline $\mathrm{Si}$. This ob-

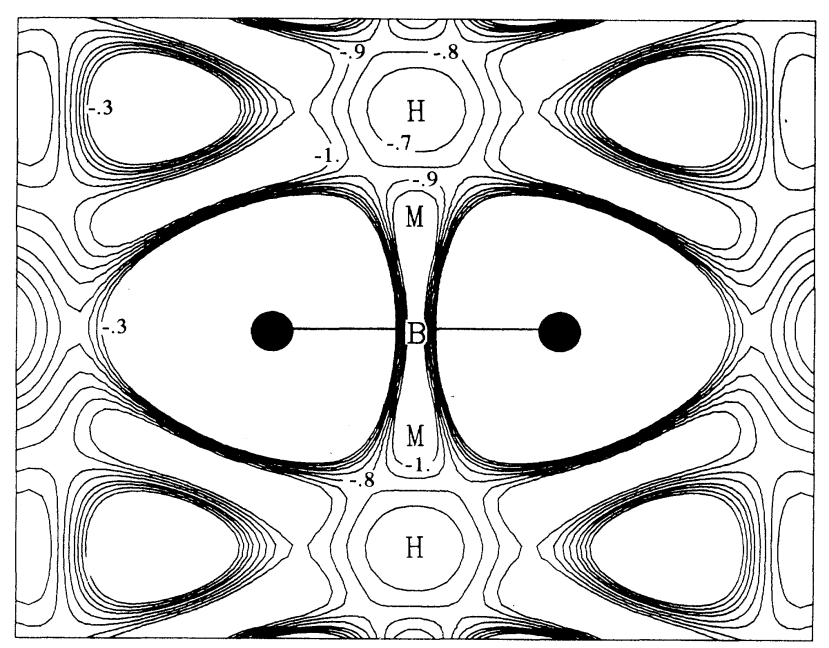

(a)

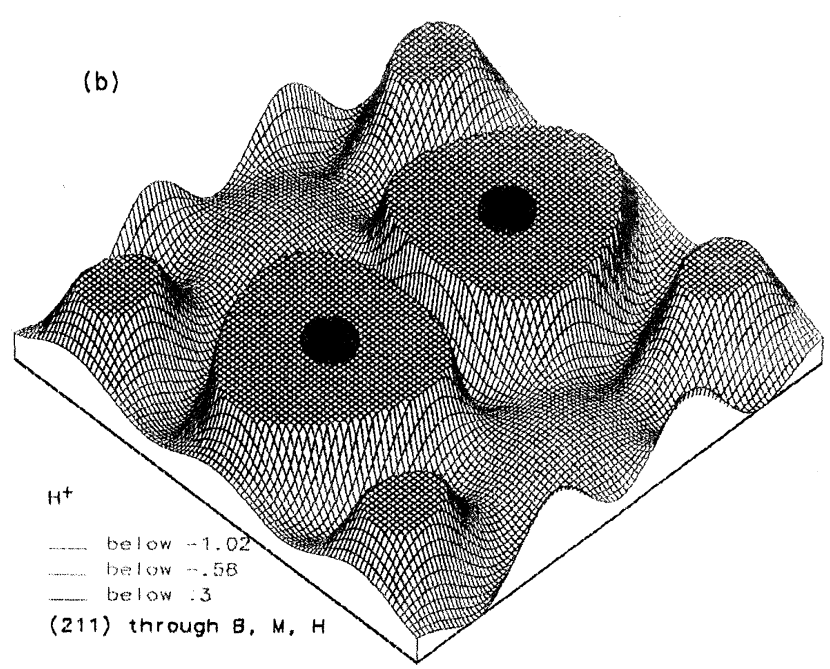

FIG. 8. (a) Contour plot and (b) perspective plot of the energy surface for $\mathrm{H}^{+}$in a (211) plane through the Si atoms, containing the sites $B, H$, and $M$. The $M$ point is located midway between a bond center and the nearest hexagonal interstitial site. The zero of energy is arbitrarily chosen at $T$ (not in the plot). See caption of Fig. 3. 
servation might be relevant for amorphous $\mathrm{Si}$, in which bond distortions are readily allowed.

\section{B. Neutral charge state}

For neutral $\mathrm{H}$ the same features and relative positions of extrema can be recognized as in the case of $\mathrm{H}^{+}$, including a global minimum at the bond center. For $\mathbf{H}$ at the bond center the neighboring $\mathrm{Si}$ atoms move out over $0.45 \AA$, to make the $\mathrm{Si}-\mathrm{H}$ distance equal to $1.63 \AA$, i.e., slightly larger than in the positive charge state. The second-neighbor relaxation is the same as for the positive charge state. The energy cost due to $\mathrm{Si}$ motion, as described at the end of Sec. III A, is $1.73 \mathrm{eV}$ for the case of the relaxations appropriate for neutral $\mathbf{H}$. As in the case of $\mathrm{H}^{+}$, we have examined carefully whether there is any tendency for $\mathrm{H}^{0}$ to preferentially bind to one of the Si neighbors, leading to an asymmetric configuration, as suggested by DeLeo et al. ${ }^{46}$ In contrast to Ref. 46, we find that the symmetric situation is lowest in energy. The saddle point of the migration path in the (110) plane is again located on the line between $C$ and $T$, but closer to $T$ than in the case of $\mathrm{H}^{+}: \mathrm{H}$ is $0.60 \AA$ away from $T$ now. Relaxation of the $\mathrm{Si}$ atoms is negligible for $\mathrm{H}$ at this site. The energy is less than $0.2 \mathrm{eV}$ higher than at the bond center. Figure 9(a) shows the charge density in a (110) plane for neutral $\mathbf{H}$ at the bond center. A concentration of charge around the impurity is immediately obvious. Most of this charge in the bond region is related to $\mathrm{H}$ induced levels buried in the valence band. It is interesting to also examine the spin density which results from a spin-polarized calculation, as described in Sec. II E. Figure 9(b) shows the difference between the spin-up and spin-down densities. This figure is remarkably similar to one that would result from plotting the charge density associated with the $\mathrm{H}$-induced defect level in the band gap (this being the level that is occupied with one, e.g., spinup, electron in the neutral charge state). It is clear that this density corresponds to an antibonding combination of Si orbitals, with mainly $p$-type character. Notice that virtually no spin density is to be found at the bond center itself. These observations can be relevant for interpretation of muon-spin-resonance experiments. ${ }^{19}$

The charge density for neutral $\mathbf{H}$ at the $T$ site is shown in Fig. 10(a). Note that the $T$ site is not a stable site for $\mathrm{H}^{0}$ in $\mathrm{Si}$, but it is educational to inspect Fig. 10 and compare it with Fig. 9. The difference between spin-up and spin-down densities is displayed in Fig. 10(b). Once again, it corresponds closely to the density associated with the $\mathbf{H}$-induced defect level, which is now below the top of the valence band. This density is now clearly associated with'an $s$-like state centered on the impurity.

Turning back to the energy surface for $\mathrm{H}^{0}$, we note that the path through the region of high electron density is favored (as for $\mathrm{H}^{+}$), but the low-density path is only $0.2 \mathrm{eV}$ higher. Thus, neutral $\mathbf{H}$ seems to be able to move rather freely through the network with very small energy barriers. We note that the $T$ site is a local maximum of the energy surface for $\mathbf{H}^{0}$. Moving from $T$ towards a substitutional site, the energy first decreases and then in- creases in the [111] direction. However, the lowest energy in this antibonding direction (less than $0.1 \mathrm{eV}$ lower than at $T$ ) does not correspond to a local minimum, but to a saddle point, i.e., the energy can be lowered by moving the $\mathbf{H}$ off the [111] direction. The same conclusion holds for the hexagonal interstitial $(H)$ site, which lies in
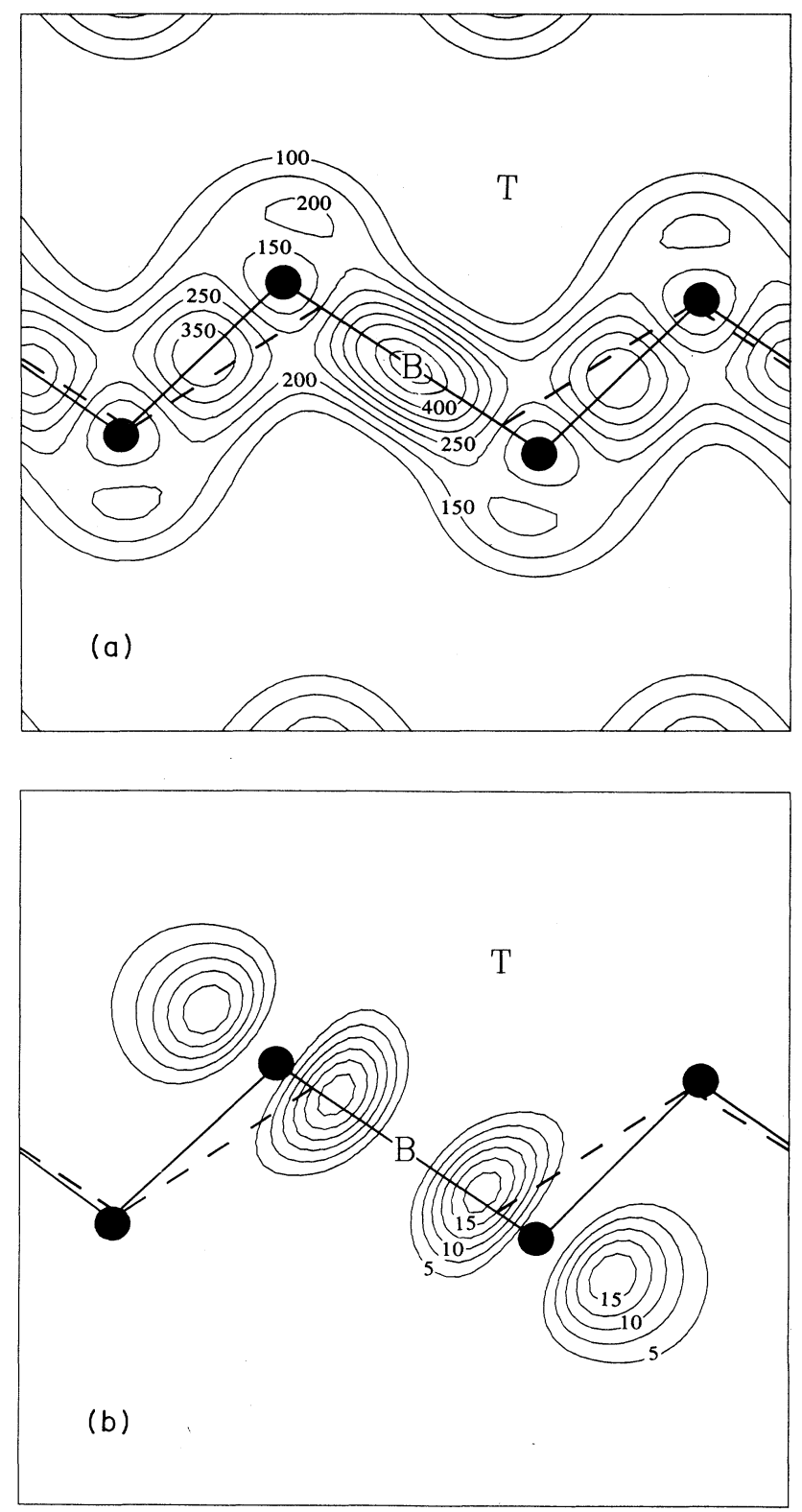

FIG. 9. (a) Contour plot of the charge density in the (110) plane through the atoms for neutral $\mathbf{H}$ at the bond center. The $\mathrm{Si}$ atoms in their relaxed positions are indicated with black dots and connected with solid lines. Dashed lines connect the unrelaxed atomic positions. The contour interval is 50; units are electrons per unit cell (for a supercell containing one $\mathrm{H}$ and 32 $\mathrm{Si}$ atoms). (b) Contour plot of the difference between spin-up and spin-down densities in the (110) plane through the atoms for neutral $\mathrm{H}$ at the bond center. The contour interval is 2.5 electrons/(unit cell). 
the [111] direction halfway between two $T$ sites. The $H$ site is a local minimum along the [111] direction, but only a saddle point when considered in three dimensions. Similar conclusions regarding antibonding and hexagonal sites hold for the positive charge state, where the energy differences are more pronounced. It is interesting to note
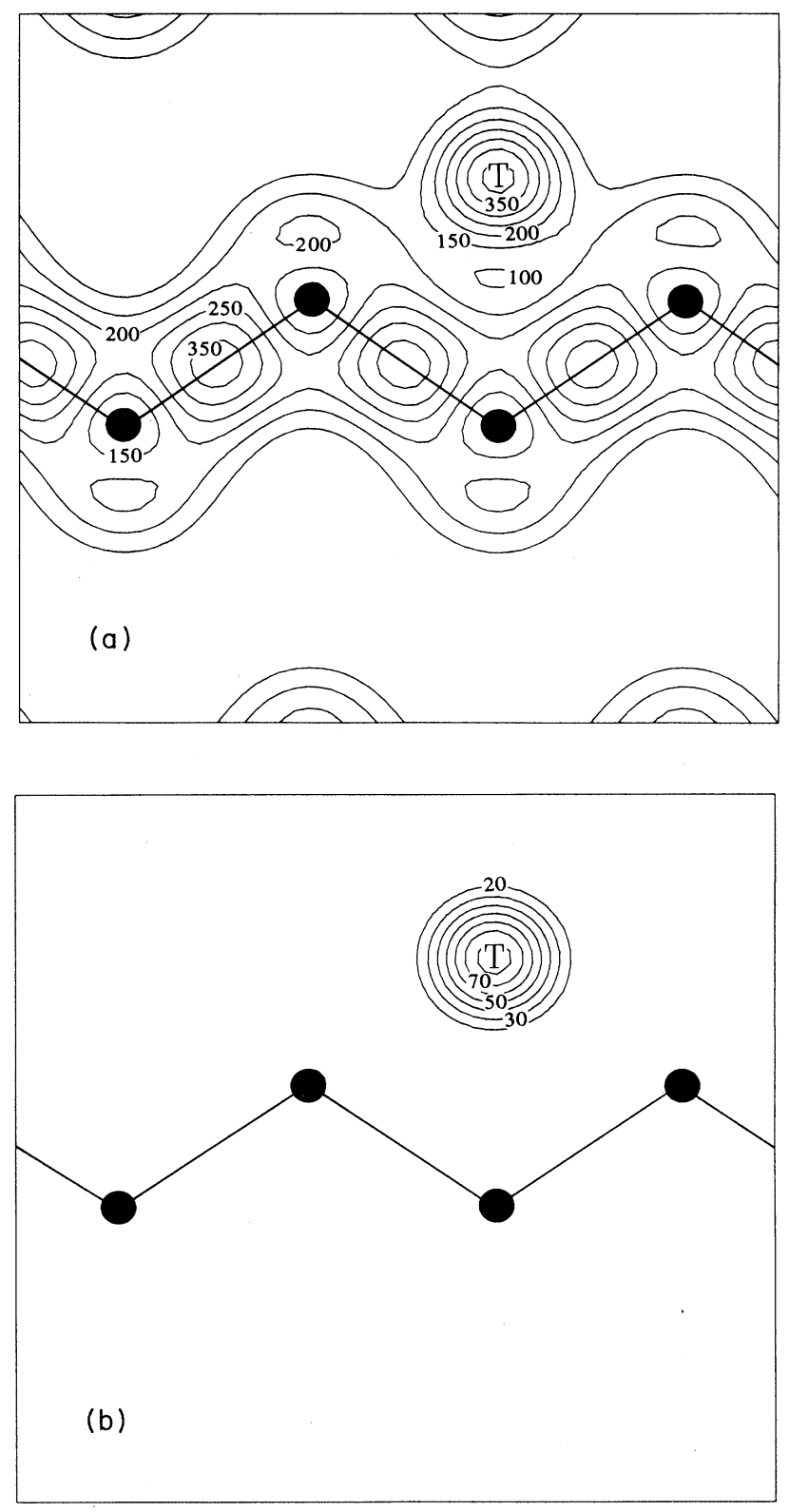

FIG. 10. (a) Contour plot of the charge density in the (110) plane through the atoms for neutral $\mathrm{H}$ at $T$. The $\mathrm{Si}$ atoms are indicated with black dots; no relaxation occurs. The contour interval is 50; units are electrons per unit cell (for a supercell containing one $\mathrm{H}$ and $32 \mathrm{Si}$ atoms). (b) Contour plot of the difference between spin-up and spin-down densities in the (110) plane through the atoms for neutral $\mathrm{H}$ at $T$. The contour interval is 10 electrons/(unit cell). that the instability of the antibonding site also occurs for $\mathrm{H}$ around a boron acceptor in $\mathrm{Si}^{47}$ eliminating this site as a candidate for the structure of $\mathrm{B}-\mathrm{H}$ complexes that result from passivation (see further).

\section{Negative charge state}

The negative charge state distinctly differs from $\mathrm{H}^{+}$ and $\mathrm{H}^{0}$ in that it is now the low-electron-density regions of the crystal which provide the most stable sites for the impurity. This can be understood by realizing that the energy cost of placing a second electron in the level in the gap (which was the trademark of the high-density sites) becomes too high, and it is more favorable to move the $\mathrm{H}$ to locations where the induced defect level occurs at lower energies. The $T$ site is now the lowest in energy, with the energy rising sharply outside the low-density regions. In particular, the $B$ site is now more than $0.5 \mathrm{eV}$ higher in energy than the $T$ site. The barrier for migration along a path through the low-density region and going through the hexagonal interstitial site is $0.25 \mathrm{eV}$.

The negative charge state is thus the only one for which the $T$ site is a stable site (local and global minimum in the energy surface). The charge density associated with this state is quite similar to that depicted in Fig. 10. This is the position for which the analysis of Altarelli and Hsu applies, showing why the $\mathrm{H}$ level is expected to be deep and not effective-mass-like. ${ }^{48}$

\section{Relative stability of different charge states}

We now examine the relative energies of the different charge states, in order to determine the lowest-energy state. To alter the charge state, electrons must be taken from or removed to a reservoir; the Fermi level determines the energy of electrons in this reservoir. The relative energies therefore depend on the position of the Fermi level. Figure 11 shows the relative formation energies for different charge states, as a function of Fermi-level position. To simplify the plot, we only show the formation energies for the impurity positions which correspond to the global minimum for a particular charge state, i.e., $B$ for $\mathrm{H}^{+}$and $\mathrm{H}^{0}$, and $T$ for $\mathrm{H}^{-}$. Figure 11(a) shows the values directly obtained from the LDA calculations. As pointed out above, these suffer from an uncertainty in the position of the defect level. Rigorous calculational schemes which could eliminate these uncertainties by going beyond the LDA are presently prohibitively complex and too computationally demanding to apply to defect calculations. We have therefore applied a very simple $a$ posteriori correction, amounting to a rigid shift of the defect level together with the conduction bands, to bring the band gap into agreement with experiment. The result of this procedure is shown in Fig. 11(b). The energies are shifted now, according to the number of electrons present in the level. We stress that Fig. 11 is not intended to display quantitative results, but merely to provide a qualitative indication of the stability of different charge states.

In $p$-type material (Fermi level at the top of the valence band), the lowest-energy state is $\mathrm{H}^{+}$in the high-density region; thus, $\mathrm{H}^{+}$diffuses via the high-density path and exhibits donorlike behavior. These conclusions are 
unambiguous and independent of any error bars in our LDA calculations. This result confirms the suggestion that the passivation of $p$-type material is a direct result of compensation, i.e., electrons from neutral $\mathrm{H}$ atoms annihilate the free holes in the valence band. ${ }^{16}$ Pairing between $\mathrm{H}^{+}$and ionized acceptors follows compensation. The structure of the hydrogen-impurity complexes that result from this pairing will be addressed in a forthcoming publication. ${ }^{47}$

From Fig. 11 we see that our calculations predict $\mathbf{H}$ to be a negative- $U$ impurity, much like the $\mathrm{Si}$ selfinterstitial. ${ }^{49}$ In $p$-type material the stable state is $\mathrm{H}^{+}$in the high-density region; as the Fermi level is raised, however, the stable state becomes $\mathrm{H}^{-}$in the low-density region. $\mathbf{H}^{0}$ is not the stable state for any Fermi level. However, the uncertainty in the LDA energy levels (and in our simple correction procedure) makes the error bar too large to unambiguously exclude the occurrence of $\mathbf{H}^{0}$.

\section{E. Vibrational frequencies}

The frequencies of the hydrogen stretching mode for $\mathrm{H}^{+}$and $\mathbf{H}^{0}$ at the bond-center site are calculated in a 32 -
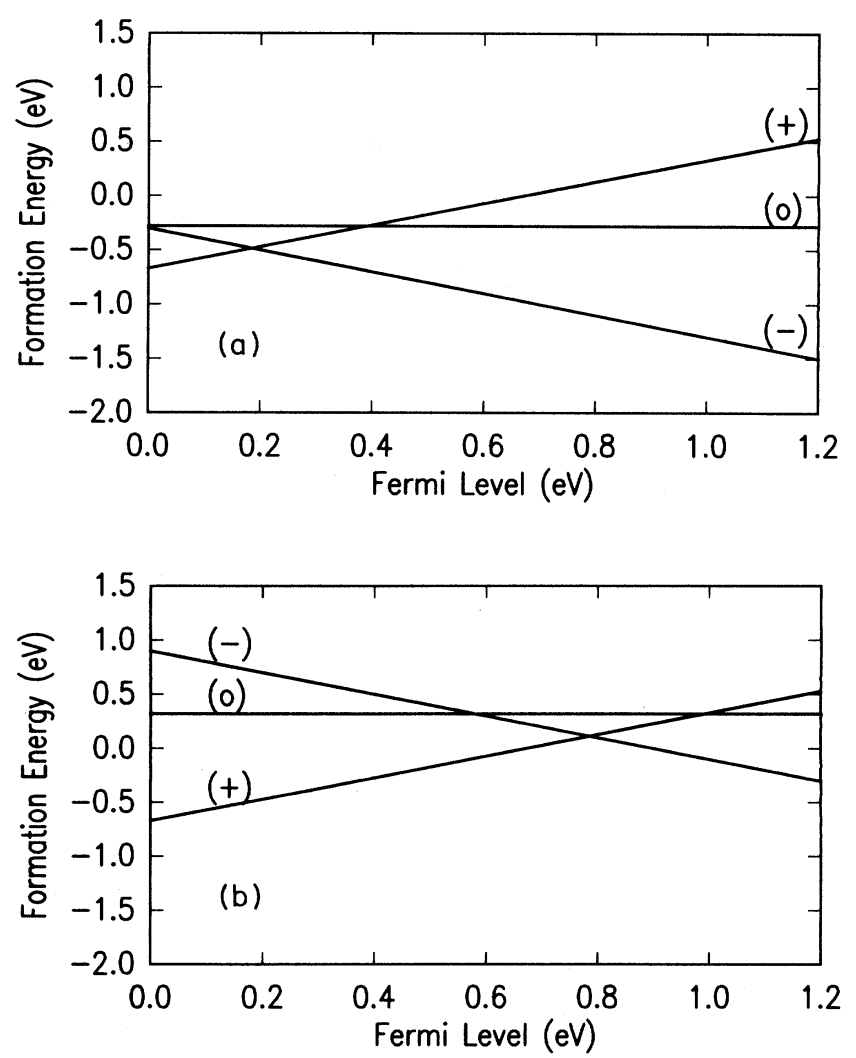

FIG. 11. Relative formation energies for different charge states of a $\mathbf{H}$ interstitial impurity in $\mathrm{Si}$. (a) shows the straight LDA values, while (b) results from applying a simple correction scheme to the energy levels (see text). The zero of energy is arbitrarily chosen as the energy of $\mathrm{H}^{0}$ at $T$. This figure is not intended to display quantitative results, but merely to provide a qualitative indication of the stability of different charge states. atom cell at a cutoff of $(10 ; 20) \mathrm{Ry}$. The $\mathbf{H}$ atom is moved from the equilibrium position in the [111] direction (towards the $\mathrm{Si}$ atoms) over distances of $2 \%$ and $4 \%$ of the $\mathrm{Si}-\mathrm{Si}$ equilibrium bond length. Since the proton is much lighter than the $\mathrm{Si}$ atoms, it is a good approximation to assume that the $\mathrm{Si}$ atoms do not move on the time scale of the $\mathbf{H}$ vibration. Relaxation of the host crystal is therefore kept fixed to that of the equilibrium position. The energy differences obtained from the calculations for different $\mathbf{H}$ positions are fitted to a parabola, from which the vibrational frequency can be determined. This procedure leads to $2210 \mathrm{~cm}^{-1}$ for $\mathbf{H}^{+}$and $1945 \mathrm{~cm}^{-1}$ for $\mathbf{H}^{0}$. The error bar on these values is $\pm 100 \mathrm{~cm}^{-1}$. Experimental values ${ }^{50,51}$ for stretching modes involving a single $\mathbf{H}$ atom in hydrogenated amorphous or crystalline Si range between 2000 and $2200 \mathrm{~cm}^{-1}$. It has often implicitly been assumed that such stretching modes involve single $\mathrm{Si}-\mathrm{H}$ bonds (such as for $\mathbf{H}$ tying off a dangling bond at a vacancy). The present results show, however, that bondcentered $\mathbf{H}$ in crystalline $\mathrm{Si}$ gives rise to similar frequencies.

\section{F. Discussion}

A large amount of experimental information has been accumulated in recent years based on observations of interactions of $\mathbf{H}$ with shallow impurities. Interpretations of the data were often based on contradicting assumptions, as pointed out by Pantelides. ${ }^{16}$ The comprehensive theoretical description provided in the present study now allows a coherent interpretation of all the data. We will also discuss results from experiments which directly address the problem of the location of $\mathbf{H}$ (or muonium) in the Si crystal.

\section{Passivation of shallow impurities}

It is known that the final result of the passivation mechanism in $p$-type material is the formation of neutral acceptor-H pairs, as observed in infrared spectroscopy measurements, ${ }^{3,4,52}$ Raman studies, ${ }^{53}$ and ion-channeling measurements. ${ }^{54,55}$ The structure of these pairs will be addressed in a separate publication. ${ }^{47}$ In order to understand the formation of these $\mathrm{H}$-acceptor pairs, however, one needs to know the nature of the charge states of $\mathbf{H}$ along its diffusion path, which will determine which hydrogen-impurity reactions can occur. The assumptions that had been made previously were often contradicting and mutually inconsistent. Pantelides ${ }^{16}$ showed that the only way to account for all the available data in $p$-type material was for $\mathrm{H}$ to have a deep donor level in the band gap. This conclusion has now been confirmed by the present theoretical results.

Hydrogen atoms in $p$-type material (where the Fermi level is below the hydrogen's donor level) prefer the positive charge state and will lose their electrons; these electrons can annihilate the holes through a mechanism of direct compensation. Pairing of hydrogen with acceptors is not necessary for compensation and passivation of $p$ type material, as has clearly been shown in recent experiments by Johnson and Herring. ${ }^{56}$ Once $\mathrm{H}^{+}$has been formed, however, its high mobility and Coulombic attrac- 
tion to negatively charged acceptor impurities will readily lead to the formation of acceptor-hydrogen pairs: $\mathbf{H}^{+}+\mathbf{B}^{-} \rightarrow(\mathbf{H B})^{0}$ (where boron has been chosen as a typical acceptor). The pair formation is therefore a consequence of passivation in $p$-type material.

The term compensation is often presumed to imply that the stable state of the system is such that the atoms which act as donors are spatially separated from the acceptors which they compensate. This occurs, for instance, in the case of compensation by counterdoping, e.g., adding phosphorus to boron-doped Si. The term compensation, however, in general applies to any situation in which electrons from donor atoms annihilate free holes, and as such correctly describes the hydrogenation of $p$-type material. Because of the high mobility of the $\mathrm{H}$ species, the final experimentally observed situation will usually be such that $\mathrm{H}$ is paired with acceptors; pairing is only absent during the initial (transient) phase of hydrogenation, or at a temperature sufficiently high to dissociate $\mathrm{H}$-acceptor pairs. Under those conditions, compensation is the accurate description for the state of the system. Our major goal in stressing the compensation aspect is to make clear that pairing is not essential for passivation, and that, indeed, the reaction of pair formation can only be correctly understood if compensation is considered to be the initial step. It should be clear that calculations in which only the structure of the resulting $\mathrm{H}$ acceptor pairs is addressed cannot have any bearing on the issue of compensation as the initial step in the passivation mechanism. The statements by Chang and Chadi, ${ }^{57}$ claiming that compensation is not involved in the passivation, are therefore unfounded, since they are inferred solely from an analysis of the already formed $\mathrm{H}$ B pair.

The sequence of events in which pair formation follows compensation is essential for understanding a wide variety of experimental results, which will be summarized below.

(1) Since the diffusing species in $p$-type material is positively charged, electric fields are expected to significantly influence the diffusion properties. The observed electric field dependence ${ }^{7,9}$ of hydrogen neutralization of shallow acceptors follows immediately, without having to invoke participation of free holes in the reaction. ${ }^{7}$

(2) When the $p$-type (B-doped) material is counterdoped, making it effectively $n$ type, $\mathbf{H}$ diffusion is retarded and $\mathrm{H}$-acceptor pairing is suppressed; the final $\mathrm{H}$ concentration is 2 orders of magnitude smaller than in $p$ type. ${ }^{4}$ Our theory shows indeed that if the Fermi level is raised the $\mathrm{H}^{+}$concentration will decrease. Neutral or negative $\mathbf{H}$ will not react with $\mathrm{B}^{-}$the way $\mathbf{H}^{+}$does, and the final concentration of $(\mathrm{BH})$ pairs will be significantly lower. A thin $n$-type overlayer was also observed to block the penetration of hydrogen. ${ }^{7,8}$ Once again, no $\mathrm{H}^{+}$ can be formed in this layer. If $\mathrm{H}^{-}$is formed, it is kept out of the $p$-type substrate by the electric field in the depletion region. If $\mathrm{H}^{0}$ would be formed, it would not as readily pair up with $\mathrm{B}^{-}$as $\mathrm{H}^{+}$does.

(3) Reverse bias of the junction formed by an $n^{+}$-type overlayer on a $p$-type substrate during hydrogenation results in a suppression of neutralization in the space- charge layer. The actual experiments were carried out with deuterium, an isotope of hydrogen which is more readily detectable with secondary-ion mass spectrometry. ${ }^{7}$ The concentration of deuterium in the spacecharge layer can greatly exceed the boron concentration, without neutralization occurring. These observations are consistent with molecule formation in that region, and a suppression of $(\mathrm{BH})$ pairing due to the absence of $\mathrm{H}^{+}$. Similar results from experiments by Tavendale $^{9}$ were explained as due to field drift of a positively charged species under an electric field. The fact that this positively charged species is $\mathrm{H}^{+}$(and not free holes) has recently been unambiguously established by Johnson and Herring, ${ }^{56,58}$ who carefully analyzed the variation with depth of the $\mathrm{H}$ concentration in $p-n$ junctions. Their results show that $\mathrm{H}$ must have a deep donor level, not far from midgap.

(4) Recent experiments by Johnson and Herring ${ }^{56}$ have also provided direct support for the compensation mechanism. By carrying out electrical measurements in real time during hydrogenation they were able to directly study the migrating species, rather than having to infer its properties from formation kinetics of various $\mathbf{H}$ related complexes. At $300^{\circ} \mathrm{C}$ a temperature at which any $(\mathrm{HB})^{0}$ complexes should be completely dissociated, they still observed a sharp increase in the resistance upon hydrogenation. These observations must be due to the indiffusion of $\mathrm{H}^{+}$and compensation.

Johnson and Herring ${ }^{59}$ have also analyzed deuterium concentrations in uniformly doped $n$-type material, as well as epitaxial layers of varying $n$-type doping on a single substrate. They concluded that $\mathbf{H}$ can occur in a negative charge state, with an acceptor level close to the donor level found in the experiments described above. While not as conclusive yet as the results for $p$-type materials, these observations do lend support to our prediction that $\mathrm{H}^{-}$is the stable charge state in $n$-type material. Further experimental work is required to test our prediction that $\mathbf{H}$ is actually a negative- $U$ impurity.

\section{Location of $H$ (and muonium) in the Si crystal}

Let us now turn to experiments in which the location of $\mathbf{H}$ in the lattice was the object of investigation. A number of ion-channeling experiments have been performed in order to determine the location of hydrogen in pure and doped Si. Once again, deuterium (D) is used, this time in order to take advantage of a nuclear reaction for detection. Picraux and Vook ${ }^{60}$ found that $D$ would be located predominantly in a single interstitial site $1.6 \AA$ along a [111] direction from a $\mathrm{Si}$ atom in the antibonding direction. A major problem of the technique is the introduction of lattice damage due to the ion beam, and the resulting attachment of $\mathrm{D}$ to these defects. The observed D positions are therefore likely not those in pure, but in damaged $\mathrm{Si}$, and may not only correspond to atomic, but also to molecular $\mathbf{H}$. This problem has been addressed in careful experiments by Nielsen, ${ }^{12}$ in which beam-induced damage was kept to a minimum. He found $80 \%$ of deuterium atoms to be located close to bond-center sites, while $20 \%$ are close to tetrahedral sites. The occurrence 
of $\mathrm{D}$ at the bond center was ruled out by Nielsen on the ground of older theoretical calculations. ${ }^{23,25,29}$ His lowtemperature results are consistent, however, with a significant fraction of D located at the bond-center site, which emerges as the lowest-energy position for $\mathrm{H}^{+}$and $\mathrm{H}^{0}$ from the present study.

As mentioned in the Introduction, a wealth of experimental information has been generated from muon-spinresonance experiments. Our results for the behavior of neutral $\mathbf{H}$ in $\mathrm{Si}$ are in general agreement with the observations on the paramagnetic center. Muonium has been found to diffuse very rapidly in $\mathrm{Si}^{61}$ in agreement with the low barriers found in our total-energy surface for $\mathbf{H}^{0}$. Recently, "anomalous muonium" has been unambiguously identified as occupying a bond center, ${ }^{20}$ in agreement with the global minimum that emerges from our calculations for $\mathbf{H}^{0}$. The so-called "normal muonium" is usually associated with the tetrahedral interstitial site. ${ }^{61}$ Our energy surface for $\mathrm{H}^{0}$ shows that the $T$ site is not a stable site. The bond-center site is the only local minimum in this surface (to an accuracy of $\sim 0.1 \mathrm{eV}$; a barrier of 0.1 $\mathrm{eV}$ would, however, be far too small to confine the muon anyway, given its large zero-point motion). However, other locations around $T$ (in the low-density region of the crystal) may account for the observed signal, with the muonium tunneling rapidly between different sites. Such sites, while not being global minima of the energy surface, are the only locations accessible to the muon which do not require the large relaxations of the $\mathrm{Si}$ host atoms necessary for a bond-center position. ${ }^{62}$ On the time scale of the muon lifetime, such relaxations may be sufficiently slow to effectively trap the muon in the low-density regions of the crystal, where relaxation of the host atoms is negligible.

These observations lead us to the following remarks. Our calculated results and energy surfaces correspond to zero temperature, and a static approximation; the mass of the particles does not enter into this description. At finite temperatures, phonon displacements of the Si atoms will create a continuously varying potential environment for the hydrogen atom; its insertion into the bond center, and diffusion along the migration paths shown above, will necessarily be coupled to the motion of the $\mathrm{Si}$ atoms. Even at zero temperature, the zero-point motion of the very light $\mathrm{H}$ atom will have significant amplitude. In principle, the total energy surfaces and information about relaxation obtained above can form the basis of an analysis in which the quantum nature of the particle is taken into account. We do not address this issue any further here.

\section{RESULTS FOR INTERACTIONS OF SEVERAL HYDROGEN ATOMS}

\section{A. $\mathrm{H}_{2}$ molecules}

First, we examine how two neutral $\mathbf{H}$ atoms may combine and form a $\mathrm{H}_{2}$ molecule in the $\mathrm{Si}$ crystal. We have found the minimum-energy position for the molecule straddling the tetrahedral interstitial site, oriented in the $\langle 100\rangle$ direction, with the atoms separated by $0.86 \AA$ (to be compared with $0.75 \AA$ in vacuum). This configuration is illustrated in Fig. 12. At the hexagonal interstitial site, which would lie on a migration path, the energy of the molecule is $1.1 \mathrm{eV}$ higher. The binding energy of $\mathrm{H}_{2}$ (as compared with isolated neutral $\mathrm{H}$ atoms at their lowest interstitial position, i.e., at the bond center) is $2 \pm 0.5 \mathrm{eV}$ per molecule, or $\sim 1 \mathrm{eV}$ per atom. This binding energy applies to the case where $\mathrm{H}_{2}$ is formed out of two isolated neutral $\mathrm{H}$ atoms. If instead the molecule were formed out of one $\mathbf{H}^{0}$ and one $\mathrm{H}^{+}$(a possibility suggested by Johnson and Herring ${ }^{58}$ ), the binding energy would be lowered by the energy difference between $\mathrm{H}^{+}$and $\mathrm{H}^{0}$. From Fig. 11, we see that in $p$-type material this difference can be up to $1 \mathrm{eV}$. If the molecule is formed out of (or dissociated into) $\mathrm{H}^{0}$ and $\mathrm{H}^{+}$, this result may explain the observation of Johnson and Herring that the binding energy of the molecule is lower than the diffusion barrier.

\section{B. H-induced defects}

Another phenomenon that involves the cooperative interaction of several $\mathbf{H}$ atoms with the Si lattice is related to the recent observation ${ }^{15,63}$ that hydrogenation can induce microdefects in a region within $\sim 1000 \AA$ from the surface. Care was taken to eliminate radiation damage that could result from direct exposure to the plasma during hydrogenation. The defects, studied with transmission electron microscopy (TEM), have the appearance of platelets along $\{111\}$ crystallographic planes, range in size from 50 to $100 \AA$, and exhibit no net Burgers vector. They cannot be categorized as intrinsic Si defects, such as dislocation loops or stacking faults. Some elastic-strain contrast was observed around the defects. The thickness of the platelets is comparable to a single $\{111\} \mathrm{Si}$ plane. By correlating the density of platelets with the deuterium concentration, one or two $\mathrm{H}$ atoms per $\mathrm{Si}-\mathrm{Si}$ bond are present. Furthermore, Raman measurements ${ }^{15}$ showed spectral features at 1960 and $2100 \mathrm{~cm}^{-1}$, which were at-

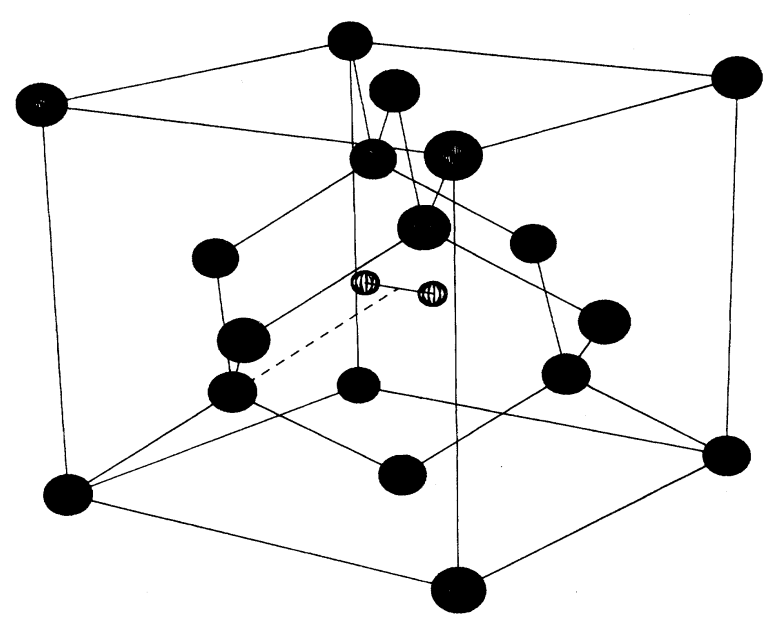

FIG. 12. Schematic illustration of the minimum-energy position of a $\mathrm{H}_{2}$ molecule in the $\mathrm{Si}$ crystal: located at the tetrahedral interstitial site and oriented along $\langle 100\rangle$. 
tributed to $\mathbf{H}$ incorporated in the Si crystal.

We have examined several possibilities (some of which were mentioned in Ref. 63) for the structure of these platelets, by performing total-energy calculations in a superlattice geometry; edge effects at the platelet boundary are thus neglected. First, we explore the situation in which one $\mathrm{H}$ atom is inserted into each of the $\mathrm{Si}-\mathrm{Si}$ bonds of a $\{111\}$ plane, as schematically illustrated in Fig. 13(a). Recalling that $\mathbf{H}$ in the bond-center position requires large relaxations of the neighboring $\mathrm{Si}$ atoms, it might be expected that the presence of $\mathbf{H}$ in a particular bond center would favor the introduction of other $\mathbf{H}$ in nearby bond centers. As discussed in Sec. III E, the vibrational frequency associated with such a bond-center configuration $\left(1945 \mathrm{~cm}^{-1}\right.$ for $\left.\mathrm{H}^{0}\right)$ is close to the experimentally observed frequencies $\left(1960\right.$ and $\left.2100 \mathrm{~cm}^{-1}\right)$; the Raman measurements alone are therefore not sufficient to exclude a model in which $\mathbf{H}$ is bonded not to one, but two $\mathrm{Si}$ atoms. However, we can eliminate this model as a candidate for the defects by inspecting the total energy. The problem is now two dimensional; we assume that no inplane relaxation occurs. The first plane of $\mathrm{Si}$ atoms near the bond center moves out over $0.45 \AA$; the second plane relaxes by $0.09 \AA$. These values are very similar to those obtained for relaxation near a single bond-centered $\mathbf{H}$. For the relaxed configuration we find that the energy per $\mathrm{H}$ is more than $0.5 \mathrm{eV}$ higher than it is for the isolated impurity, i.e., the formation of this type of extended defect is clearly unfavorable.

Another possibility for extended defect formation is the insertion of two $\mathrm{H}$ atoms in each $\mathrm{Si}-\mathrm{Si}$ bond, i.e., the formation of two $\mathrm{Si}-\mathrm{H}$ bonds out of each $\mathrm{Si}-\mathrm{Si}$ bond. It is essential to place the $\mathrm{H}$ atoms off the $\mathrm{Si}-\mathrm{Si}$ axis in order to find a favorable configuration, as illustrated in Fig. 13(b). A representative position is for the $\mathbf{H}$ atoms at two $M$ sites associated with each $\mathrm{Si}-\mathrm{Si}$ bond. The energy per $\mathrm{H}$ atom is now similar to that for isolated atoms. However, this indicates that this structure would be unstable to $\mathrm{H}_{2}$ molecule formation. We conclude that these proposed configurations are energetically not favorable.

We have therefore examined a different type of mechanism, based on the removal of $\mathrm{Si}$ atoms from the defect region, with the resulting dangling bonds tied off by $\mathbf{H}$ atoms. This mechanism is based on our calculated result that $\mathrm{H}$ atoms can assist Frenkel-pair creation. In a perfect crystal the creation of a Frenkel pair (vacancyinterstitial pair) normally costs about $8 \mathrm{eV}{ }^{64}$ If, however, a sufficient number of $\mathrm{H}$ atoms are available in the immediate neighborhood of a particular Si atom, Frenkelpair formation can actually be exothermic with a slight gain of energy. In the final configuration a self-interstitial is emitted while four $\mathrm{H}$ atoms saturate the dangling bonds of the vacancy. The calculated energy gain for the process in which a neutral interstitial $\mathrm{H}$ atom passivates a dangling bond is $\sim 2.2 \mathrm{eV}$ per $\mathrm{Si}-\mathrm{H}$ bond. ${ }^{65}$ This value is obtained by comparing the total energy of a fully saturated vacancy (i.e., four $\mathbf{H}$ atoms tying off the dangling bonds) with the sum of the energies of (a) a vacancy in which only three dangling bonds are saturated by $\mathrm{H}$, and (b) an isolated $\mathrm{H}^{0}$ at its most favorable site in the lattice. This energy value was confirmed in a superlattice calcula- tion modeling an extended defect in which a double row of $\mathrm{Si}$ atoms was removed in a $\{111\}$ plane, with all dangling bonds tied off by $\mathrm{H}$, as illustrated in Fig. 13(c). The energy gain per $\mathrm{Si}-\mathrm{H}$ bond is equal to that calculated at a single vacancy.

These theoretical results for the interaction of several $\mathrm{H}$ atoms lead us to the following conclusions. On the basis of energetic considerations, $\mathbf{H}_{2}$ molecules are the
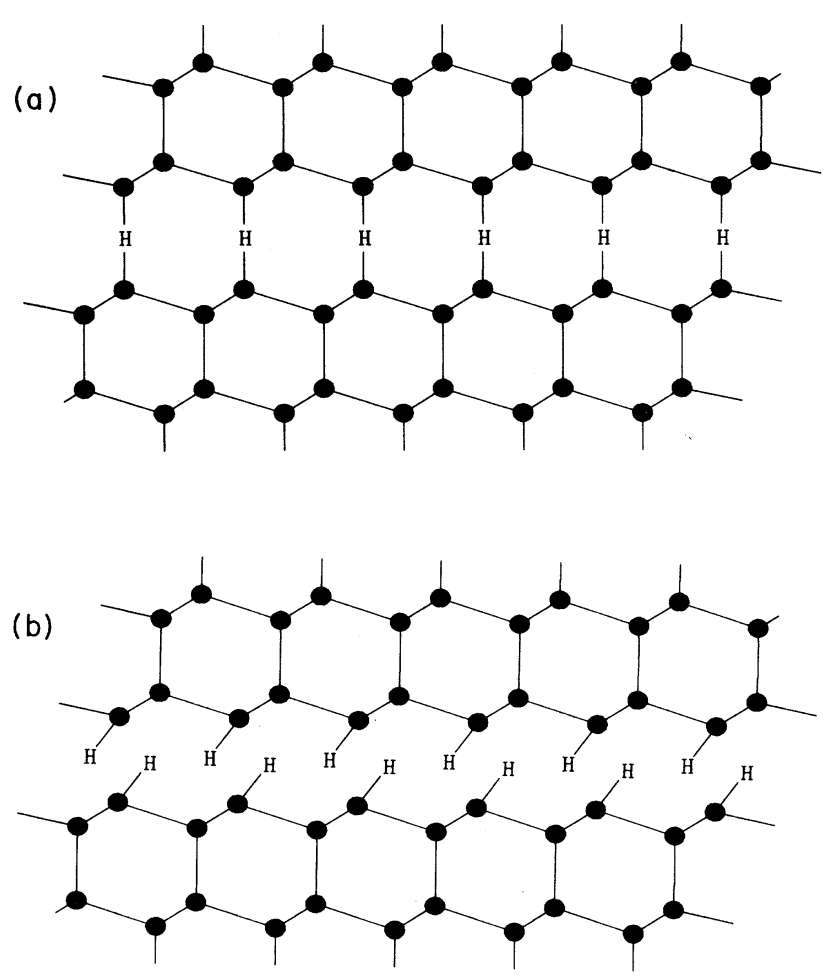

(c)
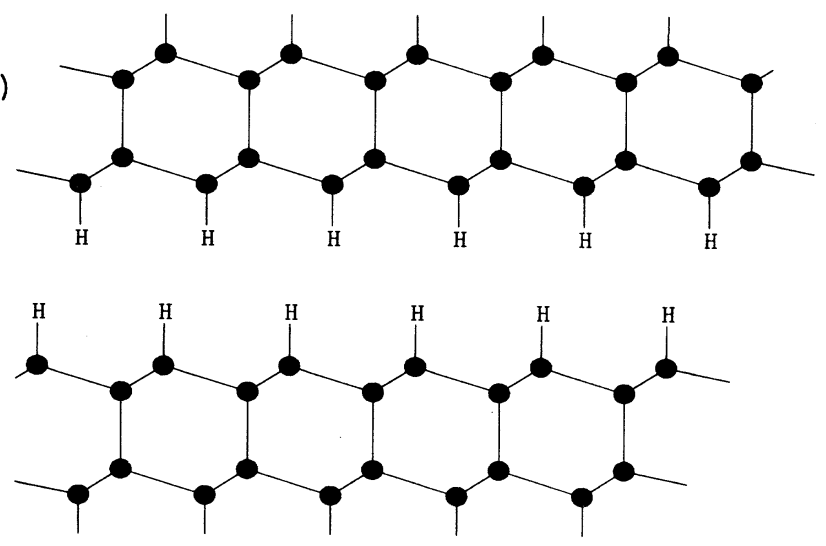

FIG. 13. Schematic illustration of possible structures for $\mathbf{H}$ induced defects ("platelets") in crystalline Si. In (a) single H atoms are situated in the bond centers of [111] $\mathrm{Si}-\mathrm{Si}$ bonds. In (b) each $\mathrm{Si}-\mathrm{Si}$ bond is replaced by two $\mathrm{Si}-\mathrm{H}$ bonds, with the $\mathrm{H}$ located off axis. In (c) a double layer of $\mathrm{Si}$ atoms has been removed through a vacancy-formation process described in the text, and the resulting dangling bonds are tied off by $\mathrm{H}$. 
preferred state for several neutral $\mathrm{H}$ atoms in pure crystalline Si. Kinetic considerations also suggest that $\mathbf{H}$ assisted Frenkel-pair creation would be a rare event. However, $\mathrm{H}$-assisted ejection of threefold- or twofoldcoordinated $\mathrm{Si}$ atoms is kinetically more favorable, such that enlargement of a preexisting defect is likely. The particular atomistic processes that lead to defect nucleation and enlargement cannot be described in more detail at this point; however, the energetic arguments given above for defect formation and extension suggest the vacancy-formation mechanism is likely to be involved in the observed hydrogen-induced damage.

\section{SUMMARY}

This work provides a comprehensive description of the diffusion and reactions of $\mathrm{H}$ in crystalline $\mathrm{Si}$, based on the first-principles pseudopotential-density-functional method. Hydrogen as an impurity shows distinctly different behavior as a function of its charge state, as exemplified by the total-energy surfaces that we generated (Figs. 3-5). $\mathbf{H}^{+}$and $\mathbf{H}^{0}$ prefer the high-electrondensity regions of the crystal, with a global energy minimum at the bond-center site. $\mathbf{H}^{-}$prefers the lowelectron-density region and has its lowest energy at the tetrahedral interstitial site. The vibrational frequencies for the $\mathrm{H}$ stretching modes at the bond center (1945 $\mathrm{cm}^{-1}$ for $\mathrm{H}^{0}$ and $2210 \mathrm{~cm}^{-1}$ for $\mathrm{H}^{+}$) are very close to measured frequencies for single $\mathrm{H}$ atoms in $a-\mathrm{Si}: \mathrm{H}$ or crystalline Si.

The stability of different charge states depends on the Fermi-level position: $\mathrm{H}^{+}$is favored in $p$-type material, providing a straightforward mechanism for passivation of $p$-type Si through compensation and subsequent pair formation. The calculations for $n$-type material produce $\mathrm{H}^{-}$ as the stable charge state, and indicate hydrogen would be a negative- $U$ impurity, but within the error bar $\mathbf{H}^{0}$ cannot be excluded.

$\mathbf{H}_{2}$ molecules are the most stable state for $\mathbf{H}$ in crystalline $\mathrm{Si}$ in the absence of other defects. Hydrogen can also induce defects; we have discussed a mechanism for extended defect formation through spontaneous Frenkelpair generation.

\section{ACKNOWLEDGMENTS}

We appreciate helpful discussions with $\mathbf{C}$. Herring, N. M. Johnson, and R. F. Kiefl. This work was supported in part by the U.S. Office of Naval Research under Contract No. N00014-84-C-0396.
*Present address: Philips Research Laboratories, 345 Scarborough Road, Briarcliff Manor, NY 10510.

${ }^{1}$ S. J. Pearton, J. W. Corbett, and T. S. Shi, Appl. Phys. A 43, 153 (1987).

${ }^{2}$ C. T. Sah, J. Y. C. Sun, and J. J. T. Tzou, Appl. Phys. Lett. 43, 204 (1983); J. Appl. Phys. 54, 5864 (1983).

${ }^{3}$ J. I. Pankove, D. E. Carlson, J. E. Berkeyheiser, and R. O. Wance, Phys. Rev. Lett. 51, 2224 (1983); J. I. Pankove, R. O. Wance, and J. E. Berkeyheiser, Appl. Phys. Lett. 45, 1100 (1984); J. I. Pankove, P. J. Zanzucchi, C. W. Magee, and G. Lucovsky, ibid. 46, 421 (1985).

${ }^{4}$ N. M. Johnson, Phys. Rev. B 31, 5525 (1985).

${ }^{5}$ N. M. Johnson, C. Herring, and D. J. Chadi, Phys. Rev. Lett. 56, 769 (1986); 59, 2116 (1987); N. M. Johnson and C. Herring, in Defects in Electronic Materials, Materials Research Society Symposia Proceedings Vol. 104, edited by M. Stavola, S. J. Pearton, and G. Davies (Materials Research Society, Pittsburgh, PA, 1988), p. 277.

${ }^{6}$ K. Bergman, M. Stavola, S. J. Pearton, and J. Lopata, Phys. Rev. B 37, 2770 (1988); in Defects in Electronic Materials, Materials Research Society Symposia Proceedings Vol. 104, edited by M. Stavola, S. J. Pearton, and G. Davies (Materials Research Society, Pittsburgh, PA, 1988), p. 281.

${ }^{7}$ N. M. Johnson, Appl. Phys. Lett. 47, 874 (1985).

${ }^{8}$ J. I. Pankove, C. W. Magee, and R. O. Wance, Appl. Phys. Lett. 47, 748 (1985).

${ }^{9}$ A. J. Tavendale, D. Alexiev, and A. A. Williams, Appl. Phys. Lett. 47, 316 (1985).

${ }^{10}$ C. H. Seager, R. A. Anderson, and J. K. G. Panitz, J. Mater. Res. 2, 96 (1987).

${ }^{11}$ A. Van Wieringen and N. Warmoltz, Physica 22, 849 (1956).

${ }^{12}$ B. Bech Nielsen, Phys. Rev. B 37, 6353 (1988).

${ }^{13}$ A. Schnegg, H. Prigge, M. Grundner, P. O. Hahn, and H. Jacob, in Defects in Electronic Materials, Materials Research
Society Symposia Proceedings Vol. 104, edited by M. Stavola, S. J. Pearton, and G. Davies (Materials Research Society, Pittsburgh, PA, 1988), p. 291; it should be noted that more recent work has indicated that impurities other than $\mathbf{H}$ may be involved in the observed phenomena.

${ }^{14}$ N. M. Johnson and M. D. Moyer, Appl. Phys. Lett. 46, 787 (1985).

${ }^{15}$ N. M. Johnson, F. A. Ponce, R. A. Street, and R. J. Nemanich, Phys. Rev. B 35, 4166 (1987).

${ }^{16}$ S. T. Pantelides, Appl. Phys. Lett. 50, 995 (1987).

${ }^{17}$ C. G. Van de Walle, Y. Bar-Yam, and S. T. Pantelides, Phys. Rev. Lett. 60, 2761 (1988).

${ }^{18}$ C. G. Van de Walle, Y. Bar-Yam, and S. T. Pantelides, in Defects in Electronic Materials, Materials Research Society Symposia Proceedings Vol. 104, edited by M. Stavola, S. J. Pearton, and G. Davies (Materials Research Society, Pittsburgh, PA, 1988), p. 253.

${ }^{19}$ B. D. Patterson, Rev. Mod. Phys. 60, 69 (1988).

${ }^{20}$ R. F. Kiefl, M. Celio, T. L. Estle, S. R. Kreitzman, G. M. Luke, T. M. Riseman, and E. J. Ansaldo, Phys. Rev. Lett. 60, 224 (1988).

${ }^{21}$ S. F. J. Cox and M. C. R. Symons, Chem. Phys. Lett. 126, 516 (1986).

${ }^{22}$ V. A. Gordeev, Yu. V. Gorelkinskii, R. F. Konopleva, N. N. Nevinnyi, Yu. V. Obukhov, and V. G. Firsov (unpublished); Yu. V. Gorelkinskii and N. N. Nevinnyi, Pis'ma Zh. Tekh. Fiz. 13, 105 (1987) [Sov. Tech. Phys. Lett. 13, 45 (1987)].

${ }^{23}$ V. A. Singh, C. Weigel, J. W. Corbett, and L. M. Roth, Phys. Status Solidi B 81, 637 (1977).

${ }^{24}$ C. O. Rodriguez, M. Jaros, and S. Brand, Solid State Commun. 31, 43 (1979).

${ }^{25}$ A. Mainwood and A. M. Stoneham, J. Phys. C 17, 2513 (1984).

${ }^{26}$ W. E. Pickett, M. L. Cohen, and C. Kittel, Phys. Rev. B 20, 
5050 (1979)

${ }^{27}$ P. J. H. Denteneer, C. G. Van de Walle, and S. T. Pantelides, Phys. Rev. Lett. 62, 1884 (1989).

${ }^{28}$ H. Katayama-Yoshida and K. Shindo, Phys. Rev. Lett. 51, 207 (1983).

${ }^{29}$ J. W. Corbett, S. N. Sahu, T. S. Shi, and L. C. Snyder, Phys. Lett. 93A, 303 (1983).

${ }^{30}$ N. Sahoo, K. C. Mishra, and T. P. Das, Hyperfine Interact. 32, 601 (1986).

${ }^{31}$ S. Estreicher, Phys. Rev. B 36, 9122 (1987).

${ }^{32}$ P. Deák. L. C. Snyder, J. L. Lindström, J. W. Corbett, S. J. Pearton, and A. J. Tavendale, Phys. Lett. A 126, 427 (1988).

${ }^{33}$ P. Deák, L. C. Snyder, R. K. Singh, and J. W. Corbett, Phys. Rev. B 36, 9612 (1987); P. Deák and L. C. Snyder, ibid. 36, 9619 (1987)

${ }^{34}$ R. M. Martin, in Festkörperprobleme (Advances in Solid State Physics), edited by P. Grosse (Vieweg, Braunschweig, 1985), Vol. XXV, p. 3 .

${ }^{35}$ P. Hohenberg and W. Kohn, Phys. Rev. 136, B864 (1964); W. Kohn and L. J. Sham, ibid. 140, A1133 (1965); exchange and correlation potentials are based on the data from D. M. Ceperley and B. J. Alder, Phys. Rev. Lett. 45, 566 (1980), as parametrized by J. Perdew and A. Zunger, Phys. Rev. B 23, 5048 (1981).

${ }^{36}$ D. R. Hamann, M. Schlüter, and C. Chiang, Phys. Rev. Lett. 43, 1494 (1979).

${ }^{37}$ J. Ihm, A. Zunger, and M. L. Cohen, J. Phys. C 12, 4409 (1979).

${ }^{38}$ A. Baldereschi, Phys. Rev. B 7, 5212 (1973); D. J. Chadi and M. L. Cohen, ibid. 8, 5747 (1973); H. J. Monkhorst and J. D. Pack, ibid. 13, 5188 (1976).

${ }^{39}$ P. J. H. Denteneer, Ph.D. thesis, Eindhoven University of Technology, 1987, available from the author upon request.

${ }^{40}$ Following the methodology of Y. Bar-Yam and J. D. Joannopoulos, Phys. Rev. B 30, 1844 (1984).

${ }^{41}$ P. O. Löwdin, J. Chem. Phys. 19, 1396 (1951).

${ }^{42}$ P. N. Keating, Phys. Rev. 145, 637 (1966); G. A. Baraff, E. O. Kane, and M. Schlüter, Phys. Rev. B 21, 5662 (1980).

${ }^{43}$ A. Zunger, in Solid State Physics, edited by H. Ehrenreich and D. Turnbull (Academic, New York, 1986), Vol. 36, pp. 275-464.

${ }^{44} \mathrm{~F}$. Beeler, Ph.D. thesis, University of Stuttgart, 1986.

${ }^{45}$ D. Vanderbilt and J. D. Joannopoulos, Phys. Rev. B 27, 6311
(1983).

${ }^{46}$ G. G. DeLeo, M. J. Dorogi, and W. B. Fowler, Phys. Rev. B 38, 7520 (1988).

${ }^{47}$ P. J. H. Denteneer, C. G. Van de Walle, and S. T. Pantelides, the following paper, Phys. Rev. B 39, 10809 (1989).

${ }^{48}$ M. Altarelli and W. Hsu, Phys. Rev. Lett. 43, 1346 (1979).

${ }^{49}$ R. Car, P. J. Kelly, A. Oshiyama, and S. T. Pantelides, Phys. Rev. Lett. 52, 1814 (1984).

${ }^{50}$ M. H. Brodsky, M. Cardona, and J. J. Cuomo, Phys. Rev. B 16, 3556 (1977).

${ }^{51}$ H. J. Stein, Phys. Rev. Lett. 43, 1030 (1979).

${ }^{52}$ M. Stavola, S. J. Pearton, J. Lopata, and W. C. DautremontSmith, Phys. Rev. B 37, 8313 (1988).

${ }^{53}$ M. Stutzmann, Phys. Rev. B 35, 5921 (1988).

${ }^{54}$ A. D. Marwick, G. S. Oehrlein, and N. M. Johnson, Phys. Rev. B 36, 4539 (1987).

${ }^{55}$ B. B. Nielsen, J. U. Andersen, and S. J. Pearton, Phys. Rev. Lett. 60, 321 (1988).

${ }^{56}$ N. M. Johnson and C. Herring, in Proceedings of the Third International Conference on Shallow Impurities in Semiconductors, Linköping, 1988, IOP Conf. Ser. (IOP, London, 1989).

${ }^{57}$ K. J. Chang and D. J. Chadi, Phys. Rev. Lett. 60, 1422 (1988).

${ }^{58}$ N. M. Johnson and C. Herring, Phys. Rev. B 38, 1581 (1988).

${ }^{59}$ N. M. Johnson and C. Herring, in Proceedings of the 15th International Conference on Defects in Semiconductors, Budapest, 1988 (Trans Tech, Aedermannsdorf, 1989).

${ }^{60}$ S. T. Picraux and F. L. Vook, Phys. Rev. B 18, 2066 (1978).

${ }^{61}$ B. D. Patterson, E. Holzschuh, R. F. Kiefl, K. W. Blazey, and T. L. Estle, Hyperfine Interact. 17-19, 599 (1984).

${ }^{62} \mathrm{G}$. D. Watkins, in Proceedings of the 15th International Conference on Defects in Semiconductors, Budapest, 1988 (Trans Tech, Aedermannsdorf, 1989).

${ }^{63}$ F. A. Ponce, N. M. Johnson, J. C. Tramontana, and J. Walker, in Proceedings of the Microscopy of Semiconducting Materials Conference, Inst. Phys. Conf. Ser. No. 87, edited by A. G. Cullis (Hilger, London, 1987), p. 49.

${ }^{64}$ Y. Bar-Yam and J. D. Joannopoulos, J. Electron. Mater. 14a, 261 (1985); R. Car, P. J. Kelly, A. Oshiyama, and S. T. Pantelides, ibid. 14a, 269 (1985).

${ }^{65}$ In contrast, J. M. Baranowski and J. Tatarkiewicz [Phys. Rev. B 35, 7450 (1987)] proposed that the interstitial $\mathrm{H}$ be put on the backside of the dangling bond. We find that the energy gain for such an arrangement is only $1.3 \mathrm{eV}$. 


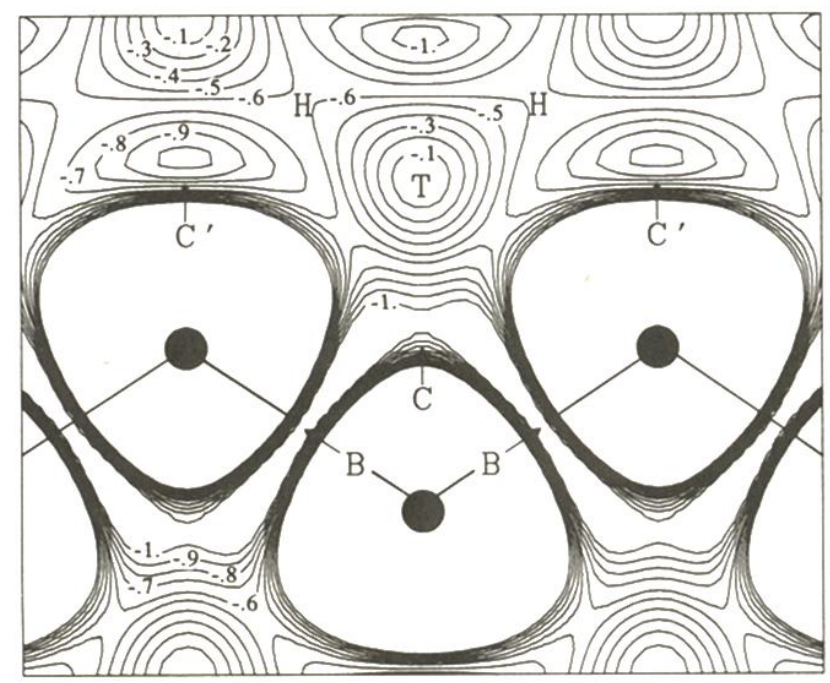

(a)

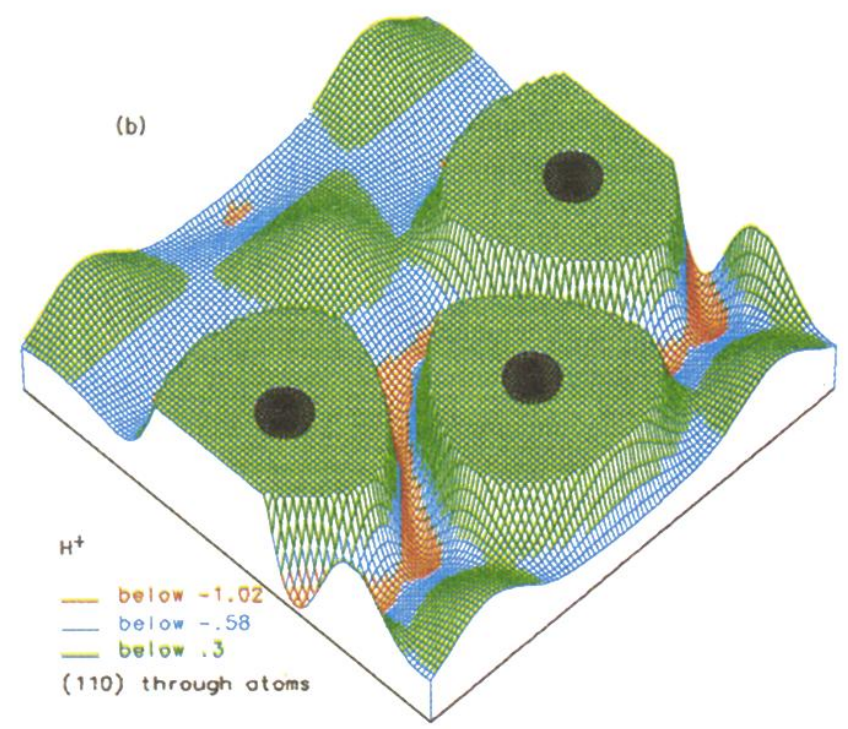

FIG. 3. (a) Contour plot and (b) perspective plot of the energy surface for $\mathrm{H}^{+}$in a (110) plane through the $\mathrm{Si}$ atoms. The zero of energy is arbitrarily chosen at $T$. The black dots represent $\mathrm{Si}$ atoms at their unrelaxed positions; the relaxations (which are different for different $\mathrm{H}$ positions) are not shown but are taken into account in the total-energy calculations. In (a) the contour interval is $0.1 \mathrm{eV}$. The color coding of the perspective plot in (b) is indicated in the figure: the energy values below $-1.02 \mathrm{eV}$ are shown in red; between -1.02 and $-0.58 \mathrm{eV}$ in blue; and between -0.58 and $0.3 \mathrm{eV}$ in green. The surface is cut off at an energy value of $0.3 \mathrm{eV}$. 


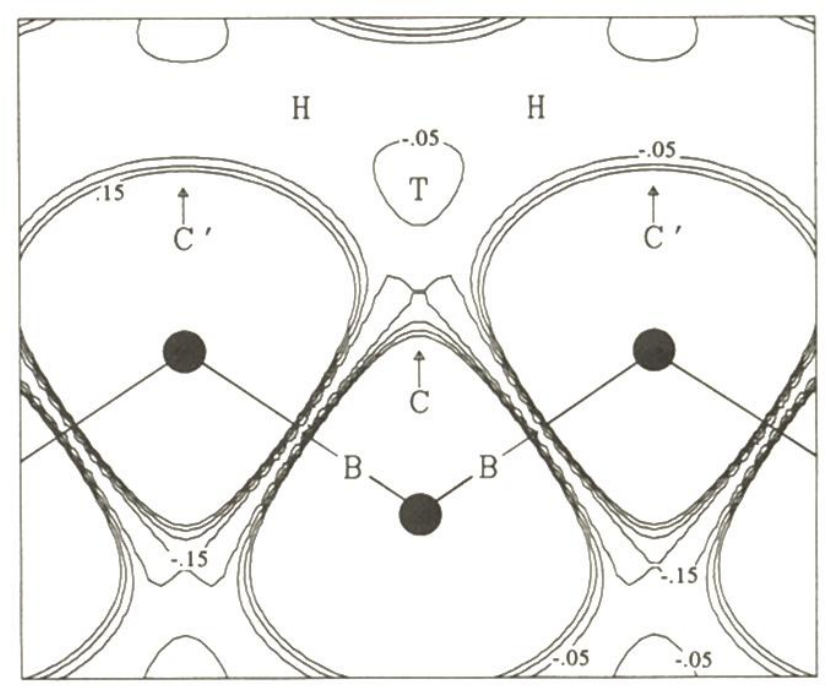

(a)

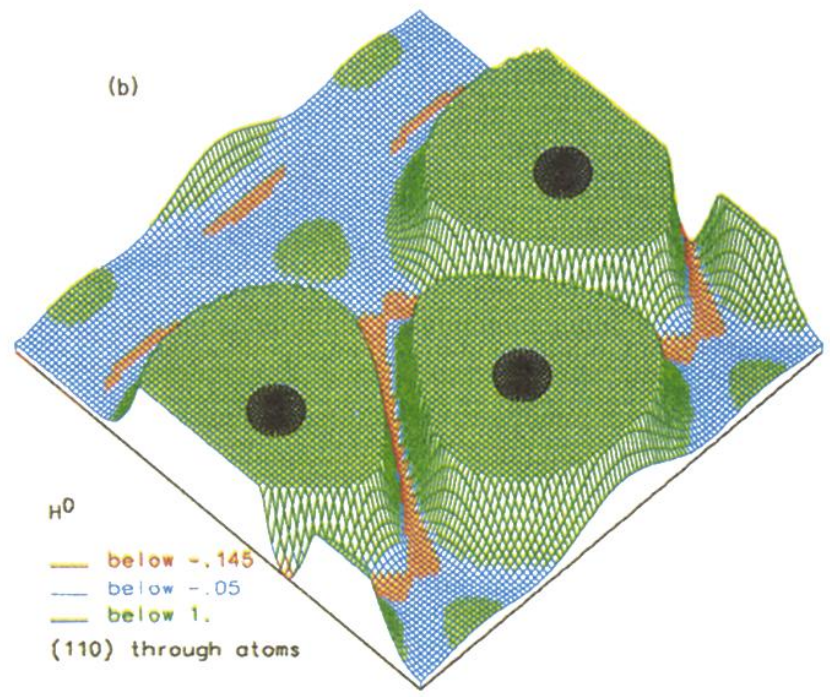

FIG. 4. (a) Contour plot and (b) perspective plot of the energy surface for $\mathrm{H}^{0}$ in a (110) plane through the $\mathrm{Si}$ atoms. See caption of Fig. 3. 


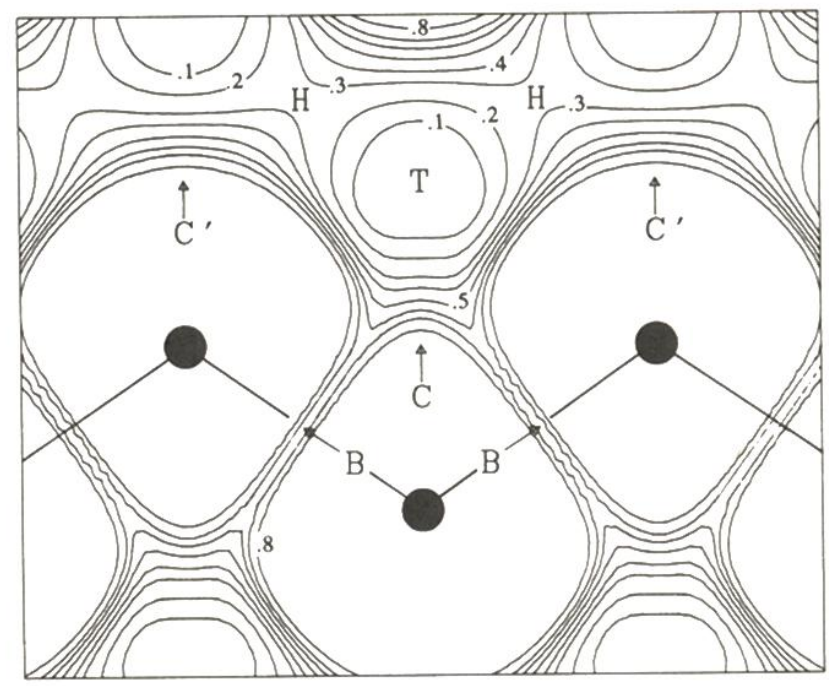

(a)

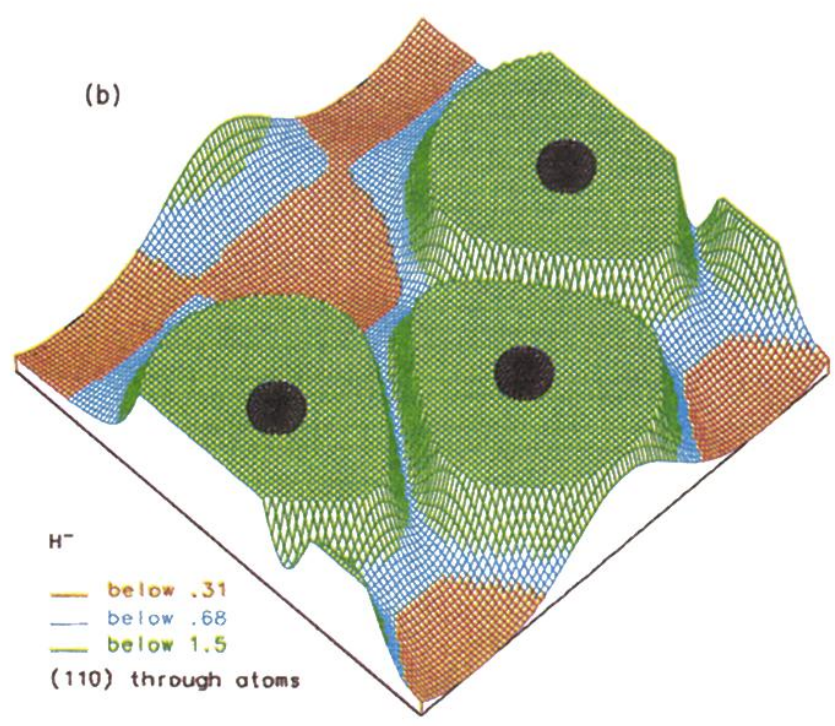

FIG. 5. (a) Contour plot and (b) perspective plot of the energy surface for $\mathrm{H}^{-}$in a (110) plane through the $\mathrm{Si}$ atoms. See caption of Fig. 3. 


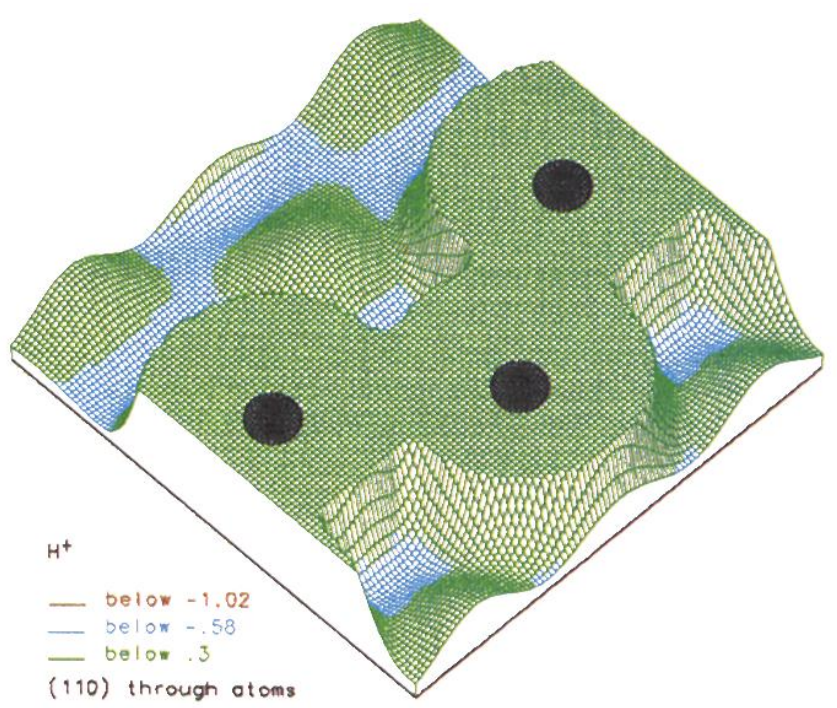

FIG. 6. Perspective plot of the energy surface for $\mathrm{H}^{+}$in a (110) plane through the $\mathrm{Si}$ atoms. To generate the values for this plot (unlike all others) the Si atoms were kept fixed in their rigid lattice positions. Comparing with Fig. 3, we see that the low-energy regions have disappeared. 


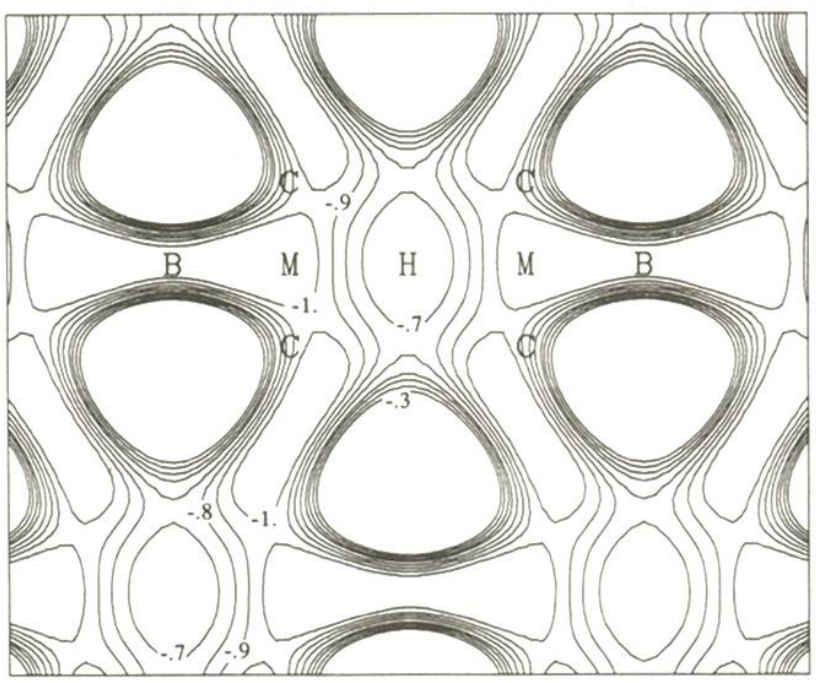

(a)

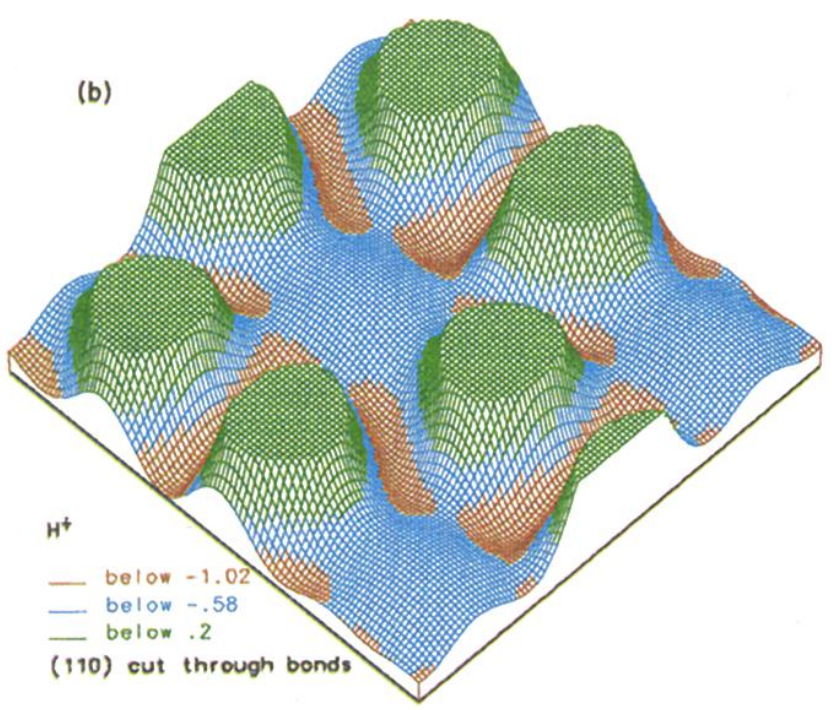

FIG. 7. (a) Contour plot and (b) perspective plot of the energy surface for $\mathrm{H}^{+}$in a (110) plane through the sites $B, C, H$, and $M$. This plane is parallel to the plane of Fig. 3, and midway between equivalent planes through the atoms. The $M$ point is located midway between a bond center and the nearest hexagonal interstitial site. The zero of energy is arbitrarily chosen at $T$ (not in the plot). See caption of Fig. 3. 


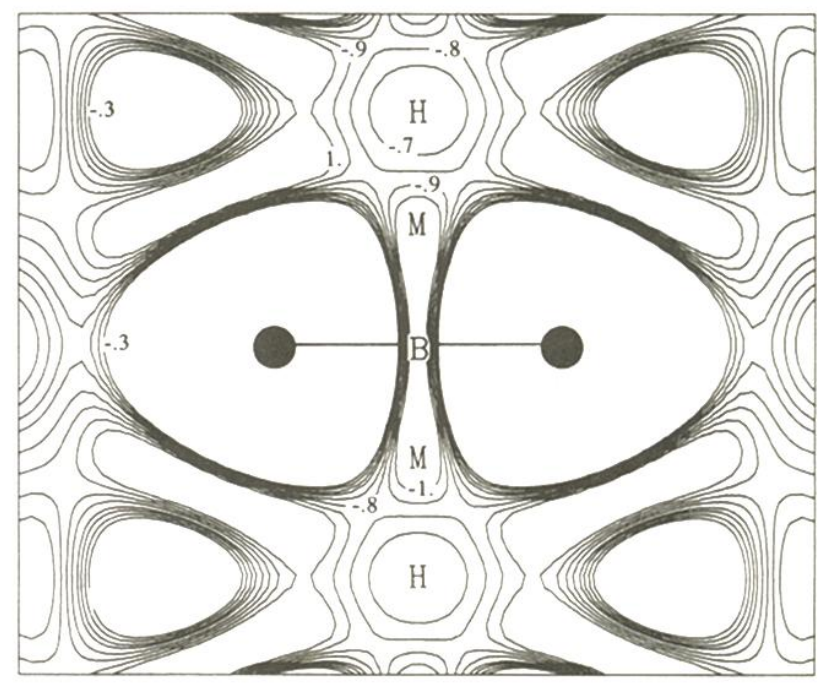

(a)

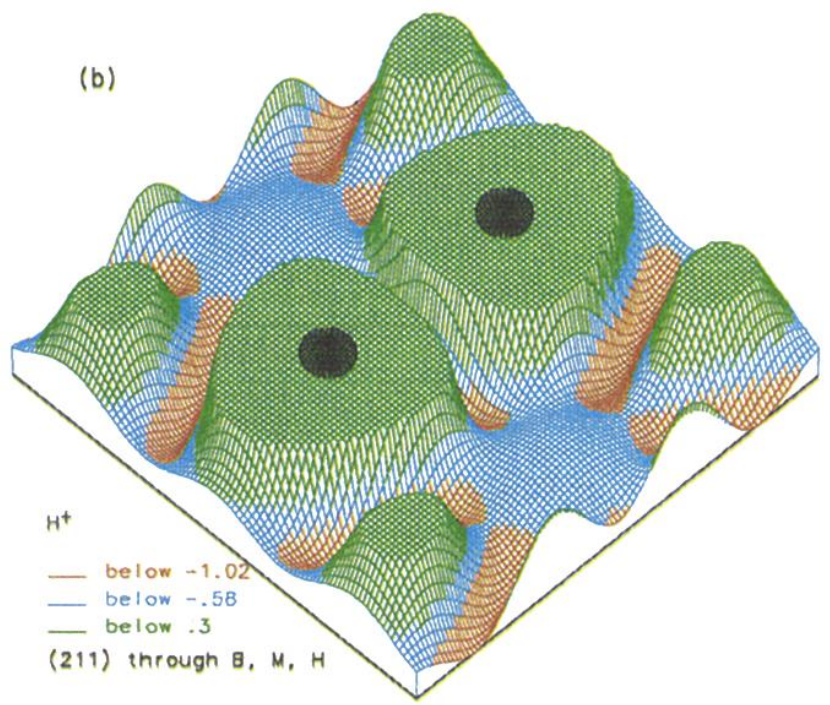

FIG. 8. (a) Contour plot and (b) perspective plot of the energy surface for $\mathrm{H}^{+}$in a (211) plane through the $\mathrm{Si}$ atoms, containing the sites $B, H$, and $M$. The $M$ point is located midway between a bond center and the nearest hexagonal interstitial site. The zero of energy is arbitrarily chosen at $T$ (not in the plot). See caption of Fig. 3. 